This is the final peer-reviewed accepted manuscript of:

Martin Huska et al 2019 Inverse Problems 35124008.

The final published version is available online at : http://dx.doi.org/10.1088/13616420/ab2d44

Rights / License:

The terms and conditions for the reuse of this version of the manuscript are specified in the publishing policy. For all terms of use and more information see the publisher's website.

This item was downloaded from IRIS Università di Bologna (https://cris.unibo.it/)

When citing, please refer to the published version. 


\title{
A CONVEX-NONCONVEX VARIATIONAL METHOD FOR THE ADDITIVE DECOMPOSITION OF FUNCTIONS ON SURFACES
}

\author{
MARTIN HUSKA ${ }^{*}$, ALESSANDRO LANZA ${ }^{\dagger}$, SERENA MORIGI ${ }^{\ddagger}$, AND IVAN SELESNICK ${ }^{\S}$
}

\begin{abstract}
We present a Convex-NonConvex variational approach for the additive decomposition of noisy scalar fields defined over triangulated surfaces into piecewise constant and smooth components. The energy functional to be minimized is defined by the weighted sum of three terms, namely an $\ell_{2}$ fidelity term for the noise component, a Tikhonov regularization term for the smooth component and a Total Variation (TV)-like non-convex term for the piecewise constant component. The last term is parametrized such that the free scalar parameter allows to tune its degree of nonconvexity and, hence, to separate the piecewise constant component more effectively than by using a classical convex TV regularizer without renouncing to convexity of the total energy functional. A method is also presented for selecting the two regularization parameters. The unique solution of the proposed variational model is determined by means of an efficient ADMM-based minimization algorithm. Numerical experiments show a nearly perfect separation of the different components.
\end{abstract}

Key words. Image decomposition, Convex non-convex strategy, ADMM, Functions on surfaces.

1. Introduction. Signal decomposition is a widely used tool in many data processing applications such as, e.g., data coding, analysis and synthesis. In such problems it is typically assumed that the observed signal is a linear mixture - or weighted average - of different source signals with specific homogeneous characteristics.

For the case of 2-D signals, Meyer in [6] proposed a variational model based on TV regularization [16] to decompose an image into two components, namely a piecewise constant - or cartoon - component and a texture - or oscillatory - component. In particular, the G norm was introduced in [6] to capture texture in noiseless images. The idea is that, while the space of functions of Bounded Variation (BV) is a good space to model cartoon images, a space close to the dual of BV is well suited to model oscillating patterns. Inspired by Meyer's work, and motivated by the difficulty of dealing with the dual of BV which is not a separable space, several variational models have been proposed to decompose gray-scale and color images [4, 1, 3, 20, 21].

Related prior work in a variational approach, proposed for image restoration rather than for image decomposition, is introduced by Chambolle and Lions in [2], where a combination of bounded variation and bounded Hessian function components is considered. More recently Gholami in [5] proposed an image restoration model based on a balanced combination of TV and Tikhonov regularization.

In this paper we address the problem of decomposing noisy scalar functions for the general case where the functions are defined over an arbitrary topology 2-manifold $\Omega$ embedded in $\mathbb{R}^{3}$ - that will be discretized by an unstructured triangle mesh and the underlying data may contain jump discontinuities that separate smoothly varying regions. In particular, we assume that the observed scalar field $b: \Omega \rightarrow \mathbb{R}$ is representable by the following three-terms additive model

$$
b=v+w+\epsilon=u+\epsilon,
$$

* Department of Mathematics, University of Bologna, Bologna, Italy. Email: martin.huska@unibo.it.

$\dagger$ Department of Mathematics, University of Bologna, Bologna, Italy. Email: alessandro.lanza2@unibo.it.

$\ddagger$ Department of Mathematics, University of Bologna, Bologna, Italy. Email: serena.morigi@unibo.it.

$\S$ Department of Electrical and Computer Engineering, New York University, Brooklyn, New York, USA. Email: selesi@nyu.edu 


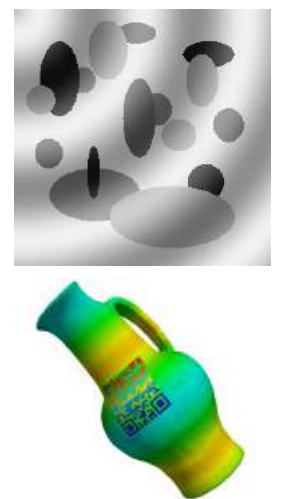

$u$
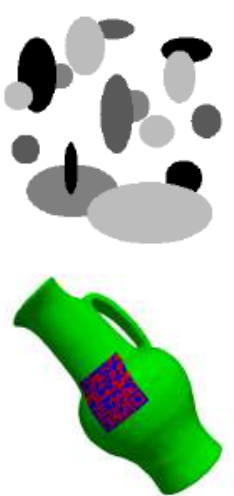

$v$
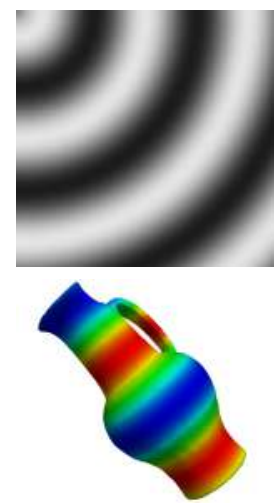

$w$

FIG. 1.1. Additive decomposition of a noise-free piecewise smooth image/scalar field $u$ into a piecewise-constant - or cartoon - component $v$ and a smooth component $w$.

with $v, w, \epsilon: \Omega \rightarrow \mathbb{R}$ the cartoon, smooth and noise components, respectively, and $u:=v+w$ the underlying noise-free piecewise smooth field. Notice that $v$ models sharp edges in $u$, whereas $w$ models the smooth part of $u$ which contains small or even large oscillations but characterized by small gradient norms, and, in practice, can capture smoothly varying intensities due, e.g., to lightning effects. In Fig. 1.1 we show an example of cartoon/smooth component decomposition of both a noiseless image (top row) and a noiseless scalar field defined over a surface in $\mathbb{R}^{3}$ (bottom row). The component $\epsilon$ in (1.1) models a noise realization, which we assume to be drawn from a (zero-mean) white Gaussian process.

In accordance with (1.1), in this paper we propose a variational model for the additive decomposition of any observed noisy scalar field $b$ into three different fields $v^{*}, w^{*}, \epsilon^{*}:=b-\left(v^{*}+w^{*}\right)$ representing estimates of the cartoon, smooth and noise components in $b$, respectively. The idea here is to use a combination of two suitable regularizers, namely a convex Tikhonov term for the smooth component and a nonconvex TV-like term for the cartoon component, together with a convex quadratic fidelity term accounting for noise. Since the noise $\epsilon$ is an oscillatory but non autocorrelated (or white) component, we consider an $\ell_{2}$ norm fidelity term rather than a $\mathrm{G}$ norm term which instead is designed to separate an oscillatory but auto-correlated (or colored) component - i.e. texture. On the other hand, the texture concept itself is not even clear in case of scalar fields sampled on unstructured triangle meshes. This even more challenging problem will be investigated in future work. The proposed variational model reads

$\left\{v^{*}, w^{*}\right\} \in \arg \min _{v, w} \mathcal{J}(v, w ; \lambda, \eta, a), \quad$ with
$\mathcal{J}(v, w ; \lambda, \eta, a):=\frac{\eta}{2} \overbrace{\int_{\Omega}\|\nabla w\|_{2}^{2} \mathrm{~d} \Omega}^{\mathcal{R}_{w}(w)}+\frac{(1-\eta)}{2} \overbrace{\int_{\Omega} \phi\left(\|\nabla v\|_{2} ; a\right) \mathrm{d} \Omega}^{\mathcal{R}_{v}(v ; a)}+\frac{\lambda}{2} \overbrace{\int_{\Omega}(v+w-b)^{2} \mathrm{~d} \Omega}^{\mathcal{F}(v, w)}$,

where $\|\cdot\|_{2}$ in (1.3) denotes the $\ell_{2}$ norm, $\nabla \cdot$ the intrinsic (Riemannian) gradient, $\lambda$, $\eta, a$ scalar model parameters satisfying

$$
\lambda>0, \quad 0<\eta<1, \quad a>0,
$$


and $\phi(\cdot ; a):[0,+\infty) \rightarrow \mathbb{R}$ a parameterized non-convex (sparsity-promoting) penalty function with properties that will be outlined in Sect. 2. In particular, the parameter $a$ allows one to tune the degree of non-convexity of $\phi(\cdot ; a)$. The domain $\Omega$ represents either a generic connected 2-manifold embedded in $\mathbb{R}^{3}$ or, as an interesting special case corresponding to the image decomposition problem, a compact rectangle in $\mathbb{R}^{2}$.

Due to term $\mathcal{R}_{v}$, the functional $\mathcal{J}$ in (1.3) is non-smooth and can be convex or non-convex depending on the parameters $\lambda, \eta, a$. In fact, as it will be illustrated in the paper, the quadratic term given by the weighted sum of the fidelity $\mathcal{F}$ and the regularizer $\mathcal{R}_{w}$ in (1.3) holds the potential for compensating non-convexity of $\mathcal{R}_{v}$.

The idea of constructing and then optimizing convex functionals containing nonconvex (sparsity-promoting) terms, referred to as Convex-NonConvex (CNC) strategy, was first introduced by Blake and Zisserman in [7], then by Nikolova in [14] for denoising of binary images and very recently by Selesnick and others for different purposes, see $[17,9,18,15,10,11,12]$ for details. Attractiveness of the CNC approach resides in its ability to promote sparsity more strongly than by using convex regularizers while at the same time maintaining convexity of the optimization problem, so that reliable convex minimization methods can be used to compute the (unique) solution.

The main contributions/proposals of this paper are summarized as follows:

- effective method for the additive decomposition of noisy scalar fields defined over triangulated surfaces into a piecewise constant and a smooth component, which, to the best of authors' knowledge, has not been proposed before;

- CNC variational model for the sought-for decomposition, with all the advantages of this kind of models; this represents a novelty also in the specific case of image decomposition;

- ADMM-based minimization algorithm which, thanks to a suitable preliminary change of variable and variable splitting procedure, allows for a very efficient computation of the (unique) model solution;

- simple strategy for an effective, automatic selection of all the parameters $\lambda, \eta, a$ of the proposed CNC model.

The paper is organized as follows. In Sect. 2 we define the non-convex sparsitypromoting penalty $\phi$ used in our model (1.2)-(1.3). In Sect. 3 we introduce the 2manifold discretization via unstructured triangular mesh and in Sect. 4 we discretize our cost functional. In Sect. 5 we give conditions for convexity of the model and in Sect. 6 we describe special model variants. Sect. 7 addresses the ADMM-based scheme for solving the proposed convex model. In Sect. 8 we discuss the automatic choice of the free parameters in the model. We present several experimental results on images and surfaces in Sect. 9 and, finally, conclusions are drawn in Sect. 10.

2. Penalty function. In the paper, we denote by $\mathbb{R}_{+}:=\{t \in \mathbb{R}: t \geq 0\}$ and $\mathbb{R}_{+}^{*}:=\{t \in \mathbb{R}: t>0\}$ the sets of non-negative and positive real numbers, respectively, by $I_{d}$ and $0_{d}, 1_{d}$ the $d \times d$ identity matrix and the $d$-entries vectors of all $0 s$ and $1 s$, and by $\left(v_{1} ; v_{2}\right)$ the column vector obtained by concatenating column vectors $v_{1}, v_{2}$.

We now introduce the penalty function used in our model (1.2)-(1.3), describe the motivations for its choice and highlight some of its properties. To fulfill our goals from both the model and the minimization algorithm perspective, $\phi$ in (1.3) must:

a) be non-convex, such that regularizer $\mathcal{R}_{v}$ can induce sparsity of gradient norms of the cartoon component more strongly than the convex TV regularizer;

b) be parameterized, such that the free parameter allows to tune the degree of non-convexity of $\mathcal{R}_{v}$ and, hence, to keep the total functional $\mathcal{J}$ convex;

c) have a range independent of its parameter, so that the degree of non-convexity 


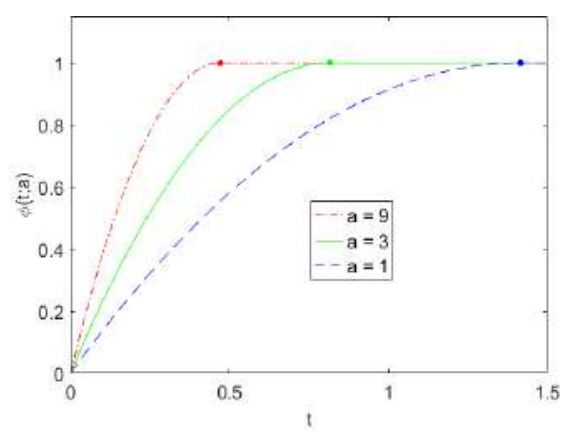

FIG. 2.1. Plots of the penalty $\phi(t ; a)$ in (2.1) for different a values. Solid dots indicate points separating, on each penalty graph, the two pieces associated with sub-domains $[0, \sqrt{2 / a}),[\sqrt{2 / a}, \infty)$.

of $\mathcal{R}_{v}$ can be tuned without changing the "weight" of $\mathcal{R}_{v}$ in the total $\mathcal{J}$;

d) have a form such that the associated multi-variate proximity operator defined by $\operatorname{prox}_{\alpha \phi}(q):=\operatorname{argmin}_{x \in \mathbb{R}^{n}}\left\{\alpha \phi\left(\|x\|_{2} ; a\right)+\|x-q\|_{2}^{2} / 2\right\}, q \in \mathbb{R}^{n}$, admits a closed-form expression which can be computed very efficiently.

In light of previous requirements, we choose a re-scaled re-parametrized version of the minimax concave penalty [22], namely the piecewise quadratic function defined by:

$$
\phi(t ; a)=\left\{\begin{array}{ll}
-\frac{a}{2} t^{2}+\sqrt{2 a} t & \text { for } t \in[0, \sqrt{2 / a}) \\
1 & \text { for } t \in[\sqrt{2 / a}, \infty)
\end{array} .\right.
$$

It is immediate to verify that the penalty above exhibits the following properties:

$$
\begin{gathered}
\phi(t ; a) \in C^{1}([0, \infty)) \cap C^{\infty}([0, \infty) \backslash\{\sqrt{2 / a}\}), \\
\phi^{\prime}(t ; a)=\left\{\begin{array}{ll}
\sqrt{2 a}-a t & \text { for } t \in[0, \sqrt{2 / a}) \\
0 & \text { for } t \in[\sqrt{2 / a}, \infty)
\end{array}, \quad \phi^{\prime \prime}(t ; a)=\left\{\begin{array}{ll}
-a & \text { for } t \in[0, \sqrt{2 / a}) \\
0 & \text { for } t \in(\sqrt{2 / a}, \infty)
\end{array} .\right.\right.
\end{gathered}
$$

In particular, we note that $a=\left|\min _{t} \phi^{\prime \prime}(t ; a)\right|, t \in[0, \infty) \backslash\{\sqrt{2 / a}\}$, such that $a$ really represents the degree of non-convexity of $\phi$ and, hence, will be referred to as the concavity parameter of $\phi$ (or, by extension, of regularizer $\mathcal{R}_{v}$ ). In Fig. 2.1 we plot $\phi(t ; a)$ for three values $a \in\{1,3,9\}$ of the concavity parameter. We remark that $\phi$ in (2.1) clearly fulfills the above outlined requirements a), b), c). In Sect. 7, devoted to the ADMM algorithm, we will prove that the proximity operator of $\phi$ admits a simple closed-form expression, such that requirement d) is also satisfied.

3. Domain discretization and gradient approximation. A connected 2manifold embedded in $\mathbb{R}^{3}$ can be approximated via a triangle mesh $\Omega:=\{V, T\}$, where $V:=\left\{X_{i}\right\}_{i=1}^{n_{V}} \in \mathbb{R}^{n_{V} \times 3}$ is the set of vertices and $T \in \mathbb{N}^{n_{T} \times 3}$ is the set of face triangles. The direct connection between two neighboring vertices $X_{i}, X_{j}$ is referred to as edge $e_{i j}$ and we define by $E:=\left\{e_{i j}\right\} \subseteq V \times V$ the set of all edges. For each vertex $X_{i}$, we denote by $\mathcal{N}\left(X_{i}\right):=\left\{X_{j}: e_{i j} \in E\right\}$ its 1-ring neighborhood, defined as the set of incident vertices to $X_{i}$, and by $\mathcal{D}\left(X_{i}\right)$ the 1-disk of adjacent triangles of the vertex $X_{i}$, with $\left|\mathcal{D}\left(X_{i}\right)\right|$ the sum of the areas of the neighboring triangles.

Under this discrete setting, a function $u: \Omega \rightarrow \mathbb{R}$ can be sampled over the vertices of the mesh and is understood as a piecewise linear function. Setting $u_{i}:=u\left(X_{i}\right)$, $i=1, \ldots, n_{V}$, the sampled function is represented by vector $u \in \mathbb{R}^{n_{V}}$. The value of 
function $u$ at a location $x$ inside a triangle $\tau=\left[X_{i}, X_{j}, X_{k}\right] \in T$ is thus given by

$$
\left.u(x)\right|_{\tau}=u_{i} \mathcal{B}_{i}(x)+u_{j} \mathcal{B}_{j}(x)+u_{k} \mathcal{B}_{k}(x)
$$

where $\left\{\mathcal{B}_{\ell}: \ell=1, \ldots, n_{V}\right\}$ represents the set of piecewise linear basis functions defined by $\mathcal{B}_{\ell}\left(X_{i}\right)=\delta_{\ell i}, \ell, i=1, \ldots, n_{V}$, which exhibit the properties of (a) local support: $\operatorname{supp}\left(\mathcal{B}_{\ell}\right)=\mathcal{D}\left(X_{\ell}\right),(\mathrm{b})$ non-negativity: $\mathcal{B}_{\ell} \geq 0$ and (c) partition of unity: $\sum_{\ell} \mathcal{B}_{\ell} \equiv 1$. By differentiating these vertex-based basis functions, we obtain a piecewise constant (face-based) tangent vector field. The intrinsic gradient of $u$ lies in the tangent space $T_{X} \Omega \subset \mathbb{R}^{3 \times n_{T}}$, and restricted to a triangle $\tau$ is thus constant and expressed as

$$
\left.\nabla u(x)\right|_{\tau}=u_{i} \nabla \mathcal{B}_{i}+u_{j} \nabla \mathcal{B}_{j}+u_{k} \nabla \mathcal{B}_{k}
$$

where the gradient of each basis function on $\tau$ is also constant and given by [19]:

$$
\nabla \mathcal{B}_{i}=\frac{X_{i}-O_{i}}{\left|X_{i}-O_{i}\right|^{2}}=\frac{1}{\left|X_{i}-O_{i}\right|} \frac{X_{i}-O_{i}}{\left|X_{i}-O_{i}\right|}=\frac{1}{\left|h_{i}\right|} \frac{h_{i}}{\left|h_{i}\right|},
$$

where $O_{i}$ is the orthogonal projection of $X_{i}$ onto the edge $e_{j k}$, thus $h_{i}$ is the triangle's height w.r.t. $X_{i}$, and $h_{i} /\left|h_{i}\right|$ is a unit vector in direction of the triangle's height. For each vertex $X_{i}$ of the triangle $\tau$ the corresponding projection $O_{i}$ can be expressed as

$$
O_{i}=X_{j}+\frac{e_{j i} \cdot e_{j k}}{\left|e_{j k}\right|} e_{j k}
$$

Using matrix-vector notation, the gradient operator (3.2) can be locally discretized and represented by matrix $G_{\tau} \in \mathbb{R}^{3 \times 3}$ and vector $u_{\tau} \in \mathbb{R}^{3}$ as follows:

$$
\left.\nabla u(x)\right|_{\tau}=G_{\tau} u_{\tau}=\left(\begin{array}{ccc}
\nabla \mathcal{B}_{i}^{1} & \nabla \mathcal{B}_{j}^{1} & \nabla \mathcal{B}_{k}^{1} \\
\nabla \mathcal{B}_{i}^{2} & \nabla \mathcal{B}_{j}^{2} & \nabla \mathcal{B}_{k}^{2} \\
\nabla \mathcal{B}_{i}^{3} & \nabla \mathcal{B}_{j}^{3} & \nabla \mathcal{B}_{k}^{3}
\end{array}\right)\left(\begin{array}{c}
u_{i} \\
u_{j} \\
u_{k}
\end{array}\right)
$$

from which the squared gradient magnitude can be written as follows

$$
\left\|\left.\nabla u(x)\right|_{\tau}\right\|_{2}^{2}=\left\langle G_{\tau} u_{\tau}, G_{\tau} u_{\tau}\right\rangle=u_{\tau}^{T} G_{\tau}^{T} G_{\tau} u_{\tau}=u_{\tau}^{T} Q_{\tau} u_{\tau},
$$

with matrix $Q_{\tau} \in \mathbb{R}^{3 \times 3}$ defined by

$$
Q_{\tau}=\left(\begin{array}{ccc}
\left\|\nabla \mathcal{B}_{i}\right\|_{2}^{2} & \left\langle\nabla \mathcal{B}_{i}, \nabla \mathcal{B}_{j}\right\rangle & \left\langle\nabla \mathcal{B}_{i}, \nabla \mathcal{B}_{k}\right\rangle \\
\left\langle\nabla \mathcal{B}_{j}, \nabla \mathcal{B}_{i}\right\rangle & \left\|\nabla \mathcal{B}_{j}\right\|_{2}^{2} & \left\langle\nabla \mathcal{B}_{j}, \nabla \mathcal{B}_{k}\right\rangle \\
\left\langle\nabla \mathcal{B}_{k}, \nabla \mathcal{B}_{i}\right\rangle & \left\langle\nabla \mathcal{B}_{k}, \nabla \mathcal{B}_{j}\right\rangle & \left\|\nabla \mathcal{B}_{k}\right\|_{2}^{2}
\end{array}\right)
$$

The matrix elements in $Q_{\tau}$ can be computed as follows

$$
\left\|\nabla \mathcal{B}_{i}\right\|_{2}^{2}=\frac{1}{\left|h_{i}\right|^{2}}, \quad\left\langle\nabla \mathcal{B}_{i}, \nabla \mathcal{B}_{j}\right\rangle=\frac{\cos \measuredangle\left(h_{i}, h_{j}\right)}{\left|h_{i}\right|\left|h_{j}\right|}
$$

and similarly for the other elements. Finally, the global discrete gradient operator is represented by matrix $D \in \mathbb{R}^{3 n_{T} \times n_{V}}$ defined by

$$
D:=\left(D_{1}^{T}, D_{2}^{T}, D_{3}^{T}\right)^{T}, \quad\left(D_{j}\right)_{i k}= \begin{cases}\nabla \mathcal{B}_{k}^{j} & \text { if } X_{k} \in \tau_{j} \\ 0 & \text { otherwise }\end{cases}
$$

where each sub-matrix $D_{j} \in \mathbb{R}^{n_{T} \times n_{V}}, j=1,2,3$, is a highly sparse matrix having $3 n_{T}$ non-zero elements. Each matrix $D_{j}$ discretizes the linear operator which simultaneously computes the $j$-th gradient component - see (3.4). 
4. CNC model discretization. To discretize (1.2)-(1.3), first we recall that the integral of a function $f$ over the whole domain $\Omega$ represented by $n_{T}$ elements (triangles/pixels) is simply the sum over all of the elements $\tau_{j}, j=1, \ldots, n_{T}$, i.e.:

$$
\int_{\Omega} f(x) \mathrm{d} \Omega=\sum_{j=1}^{n_{T}} \int_{\tau_{j}} f(x) \mathrm{d} \tau_{j} .
$$

Now we focus on a generic element $\tau$ with $d$ nodes, thus the quadrature approximates an integral via a discrete weighted sum of function values over the set of nodes

$$
\int_{\tau} f(x) \mathrm{d} \tau \approx \sum_{i=1}^{d} w_{i} f_{i}
$$

In case of piecewise constant functions $(d=1), w=s_{\tau}$, where $s_{\tau}$ represents the area of triangle $\tau$. For piecewise linear interpolants over triangle we have $d=3$ and $w_{i}=\frac{s_{\tau}}{3}, i=1,2,3$ for the three vertices, while for piecewise quadratic interpolants, one obtains a quadrature that is exact for all polynomials of degree $p \leq 3$, and the weights are: $w=27 / 60$ for the 1 centroid, $w=1 / 20$ for the 3 vertices, $w=2 / 15$ for the 3 edge midpoints. The discretization of (4.1) for piecewise linear function $f$ using (4.2) with $d=3$ leads to the exact quadrature

$$
\int_{\Omega} f(x) \mathrm{d} \Omega=\sum_{\tau=\left[X_{i}, X_{j}, X_{k}\right]} \frac{s_{\tau}}{3}\left(f_{i}+f_{j}+f_{k}\right)=\sum_{i=1}^{n_{V}} f_{i} s_{i},
$$

where we used the fact that $s_{i}=\left|\mathcal{D}\left(X_{i}\right)\right| / 3=\sum_{j \in D\left(X_{i}\right)} s_{\tau_{j}} / 3$. However, if $f$ is a quadratic function, thus the sum on the right hand side of (4.3) is only an approximation to the integral on the left.

We can thus discretize our functional $\mathcal{J}$ in (1.3) in the element-wise form:

$$
\begin{aligned}
\mathcal{J}(v, w ; \lambda, \eta, a)=\sum_{j=1}^{n_{T}}\{ & s_{\tau_{j}} \frac{\eta}{2}\left\|(D w)_{j}\right\|_{2}^{2}+s_{\tau_{j}} \frac{(1-\eta)}{2} \phi\left(\left\|(D v)_{j}\right\|_{2} ; a\right) \\
& \left.+\frac{\lambda}{2} \sum_{k=1}^{3} \frac{s_{\tau_{j}}}{d}\left(v_{k}+w_{k}-b_{k}\right)^{2}\right\}
\end{aligned}
$$

where $(D \cdot)_{j}$ represents the discrete gradient of the corresponding function at element $\tau_{j}$, and the fidelity term is rewritten in the form of element nodes. After rewriting the fidelity term in nodal form - see (4.2) - our discretized functional reads as

$$
\begin{aligned}
& \mathcal{J}(v, w ; \lambda, \eta, a)=\frac{\eta}{2} \overbrace{\left\|S^{1 / 2} D w\right\|_{2}^{2}}^{\mathcal{R}_{w}(w)}+\frac{(1-\eta)}{2} \overbrace{\sum_{j=1}^{n_{T}} s_{\tau_{j}} \phi\left(\left\|(D v)_{j}\right\|_{2} ; a\right)}^{\mathcal{R}_{v}(v ; a)} \\
& +\frac{\lambda}{2} \underbrace{\left\|S_{V}^{1 / 2}(v+w-b)\right\|_{2}^{2}}_{\mathcal{F}(v, w)}
\end{aligned}
$$

where $b, v, w \in \mathbb{R}^{n_{V}}$ are the vectors of nodal values of the associated scalar fields, the discrete gradient operator matrix $D \in \mathbb{R}^{3 n_{T} \times n_{V}}$ is defined in (3.7), and $S_{V} \in \mathbb{R}^{n_{V} \times n_{V}}$, $S \in \mathbb{R}^{3 n_{T} \times 3 n_{T}}$ are diagonal matrices defined by

$$
S_{V}=\operatorname{diag}\left(s_{1}, \ldots, s_{n_{V}}\right), \quad S=\operatorname{diag}(\widehat{S}, \widehat{S}, \widehat{S}), \quad \widehat{S}=\operatorname{diag}\left(s_{\tau_{1}}, \ldots, s_{\tau_{n_{T}}}\right) .
$$


For future reference, we introduce the real, symmetric, positive semi-definite matrix $D^{T} S D \in \mathbb{R}^{n_{V} \times n_{V}}$ which represents a discretization of the Laplace-Beltrami operator:

$$
\left(D^{T} S D\right)_{i j}= \begin{cases}\left.\sum_{\tau \in \mathcal{\mathcal { N } _ { \Delta } ( X _ { i } )}} s_{\tau}\left\|\nabla B_{i}\right\|_{2}^{2}\right|_{\tau} & \text { if } i=j \\ \left.\sum_{\tau} s_{\tau}\left\langle\nabla B_{i}, \nabla B_{j}\right\rangle\right|_{\tau} & \text { if } i \neq j \text { and } X_{i}, X_{j} \in \tau \\ 0 & \text { otherwise }\end{cases}
$$

where $\mathcal{N}_{\triangle}\left(X_{i}\right)$ denotes the 1-ring triangle neighborhood sharing the vertex $X_{i}$.

5. Analysis of the proposed discrete model. We analyze our discrete model in terms of coerciveness, convexity as well as of existence/uniqueness of solutions. To simplify notations, we introduce the total optimization variable $x:=(v ; w) \in \mathbb{R}^{2 n_{V}}$. First, in Proposition 5.1 we point out some important characteristics of $\mathcal{J}$ in (4.5)

Proposition 5.1. For any $\lambda, \eta$, a satisfying (1.4), the function $\mathcal{J}$ in (4.5) is proper, continuous, bounded from below by zero and non-coercive in $x$. In particular, $\mathcal{J}$ is constant along straight lines in its domain $\mathbb{R}^{2 n_{V}}$ of direction defined by the versor

$$
d:=\left(2 n_{V}\right)^{-1 / 2}\left(1_{n_{V}} ;-1_{n_{V}}\right) .
$$

The restriction of $\mathcal{J}$ to any feasible set defined by a linear constraint of the form

$$
\langle c, x\rangle=0, \quad c \in \mathcal{C}:=\left\{c \in \mathbb{R}^{2 n_{V}}:\langle c, d\rangle \neq 0\right\} .
$$

is coercive and, thus, $\mathcal{J}$ admits (an infinity of) global minimizers over $\mathbb{R}^{2 n_{V}}$.

Proof. It comes easily from $(2.1),(4.5)$ that $\mathcal{J}$ is proper, continuous, bounded from below by zero and constant in the direction $d$ in (5.1), thus non-coercive. In fact, it is easy to check that $\mathcal{J}(v, w)=\mathcal{J}\left(v+t 1_{n_{V}}, w-t 1_{n_{V}}\right) \forall v, w \in \mathbb{R}^{n_{V}}, \forall t \in \mathbb{R}$. The term $\mathcal{R}_{v}$ in (4.5) is bounded from above by $(1-\eta) / 2 \sum_{j} s_{\tau_{j}}$ and from below by zero, hence does not affect coerciveness of $\mathcal{J}$. The remaining quadratic term in $\mathcal{J}$, namely $\mathcal{Q}(x):=(\eta / 2) \mathcal{R}_{w}(x)+(\lambda / 2) \mathcal{F}(x)$, has Hessian $Q:=\eta L_{1}^{T} L_{1}+\lambda L_{2}^{T} L_{2}$, with $L_{1}=\operatorname{diag}\left(S^{1 / 2}, S^{1 / 2}\right) \operatorname{diag}(0, D), L_{2}=\operatorname{diag}\left(S_{V}^{1 / 2}, S_{V}^{1 / 2}\right)\left(I_{n_{V}} I_{n_{V}} ; I_{n_{V}} I_{n_{V}}\right)$. It is easy to prove that $\operatorname{null}(Q)=\operatorname{null}\left(L_{1}\right) \cap \operatorname{null}\left(L_{2}\right)=\left\{x \in \mathbb{R}^{2 n_{V}}: x=t d, t \in \mathbb{R}\right\}$, with $d$ in (5.1). Hence, $\mathcal{Q}(x)$ is coercive over any hyperplane not parallel to null $(Q)$, i.e. any feasible set defined by a linear constraint of the form in (5.1)-(5.2). This implies that the total $\mathcal{J}$ is also coercive over such sets and, thus, admits minimizers over $\mathbb{R}^{2 n_{V}}$. $\square$

In light of previous results in order to select one solution among the infinities, we equip our model with a constraint of the form in (5.1)-(5.2), i.e. we introduce the following family of linearly constrained versions of our model:

$$
x^{*} \in \underset{x \in \mathbb{R}^{2 n_{V}}}{\operatorname{argmin}} \mathcal{J}(x ; \lambda, \eta, a) \quad \text { subject to: }\langle c, x\rangle=0, c \in \mathcal{C},
$$

with $\mathcal{J}$ and $\mathcal{C}$ defined in (4.5) and (5.1)-(5.2), respectively. We remark that the constrained model in (5.3) is guaranteed to admit solutions.

5.1. Making the model (strongly) convex. We now analyze convexity of our model (5.3), namely we seek for a sufficient condition on parameters $\lambda, \eta, a$ such that $\mathcal{J}$ in $(4.5)$ is convex in $x=(v ; w)$. We notice that convexity conditions depend on the discretization choices outlined in Sections 3-4. However, the procedure below 
can be adapted to other discretization schemes. We rewrite $\mathcal{J}$ in (4.5) as follows:

$$
\begin{aligned}
\mathcal{J}(v, w ; \lambda, \eta, a) & =\underbrace{\frac{\lambda}{2}\left\|S_{V}^{1 / 2}(v+w)\right\|_{2}^{2}+\frac{\eta}{2}\left\|S^{1 / 2} D w\right\|_{2}^{2}-\frac{\gamma}{2}\left\|S^{1 / 2} D v\right\|_{2}^{2}}_{\mathcal{J}_{1}(v, w ; \lambda, \eta, \gamma)} \\
& +\underbrace{\frac{\gamma}{2}\left\|S^{1 / 2} D v\right\|_{2}^{2}+\frac{1-\eta}{2} \sum_{j=1}^{n_{T}} s_{\tau_{j}} \phi\left(\left\|(D v)_{j}\right\|_{2} ; a\right)}_{\mathcal{J}_{2}(v ; \eta, a, \gamma)}+\mathcal{A}(v, w),
\end{aligned}
$$

where $\mathcal{A}(v, w)=\lambda b^{T} S_{V}(b / 2-v-w)$ is affine in $x$ - hence, does not affect convexity and where we added and subtracted the term $(\gamma / 2)\left\|S^{1 / 2} D v\right\|_{2}^{2}$, with $\gamma>0$. Clearly, a sufficient condition for $\mathcal{J}$ to be convex is that both $\mathcal{J}_{1}$ and $\mathcal{J}_{2}$ in (5.4) are convex. In Propositions 5.2, 5.4 below we derive convexity conditions for $\mathcal{J}_{1}, \mathcal{J}_{2}$, respectively, then in Theorem 5.5 we deal with convexity of our total cost function $\mathcal{J}$ in (4.5).

Proposition 5.2. The quadratic function $\mathcal{J}_{1}$ in (5.4) is convex if and only if

$$
\gamma \leq \eta /(1+\eta \nu), \quad \nu=e_{\max } / \lambda,
$$

where $e_{\max } \in \mathbb{R}_{+}^{*}$ denotes the maximum eigenvalue of matrix $B$ defined by

$$
B=S_{V}^{-1 / 2} D^{T} S D S_{V}^{-1 / 2} \in \mathbb{R}^{n_{V} \times n_{V}},
$$

with $D, S_{V}, S$ defined in (3.7),(4.6). Moreover, if (5.5) holds strictly, $\mathcal{J}_{1}$ is strongly convex over any feasible set defined by a linear constraint of the form in (5.1)-(5.2).

The proof is postponed to the Appendix.

Lemma 5.3. Let $\phi(\cdot ; a)$ be the function defined in (2.1), $A \in \mathbb{R}^{q \times m}$ a non-zero matrix and $a, \alpha \in \mathbb{R}_{+}^{*}$ the parameters of the function $g: \mathbb{R}^{m} \rightarrow \mathbb{R}$ defined by

$$
g(y ; A, a, \alpha)=\frac{\alpha}{2}\|A y\|_{2}^{2}+\phi\left(\|A y\|_{2} ; a\right) .
$$

Then, the function $g$ is convex in $y$ if and only if

$$
a \leq \alpha .
$$

Proof. First, we notice that $g(y)=g_{1}\left(g_{2}(y)\right)$ with $g_{2}(y)=\|A y\|_{2}$ a convex function and $g_{1}(t)=(\alpha / 2) t^{2}+\phi(t ; a), t \in \mathbb{R}_{+}$, a monotonically increasing function which is convex if and only if $a \leq \alpha$. Hence, if $a \leq \alpha$ then $g$ is convex. On the other hand, if $a>\alpha$, by taking $y_{1}=0_{m}$ and $y_{2}$ such that $\left\|A y_{2}\right\|_{2}=\sqrt{2 / a}$, we have

$$
\frac{g\left(y_{1}\right)+g\left(y_{2}\right)}{2}=\frac{1}{2}\left(\frac{\alpha}{a}+1\right)<\frac{1}{2}\left(\frac{\alpha}{2 a}+\frac{3}{2}\right)=g\left(\frac{y_{1}+y_{2}}{2}\right),
$$

hence $g$ is not convex. $\square$

Proposition 5.4. Function $\mathcal{J}_{2}$ in (5.4) is convex if and only if

$$
\gamma \geq a(1-\eta) / 2 \text {. }
$$


Proof. Based on (3.4), we rewrite $\mathcal{J}_{2}$ in (5.4) in triangle-wise form:

$$
\mathcal{J}_{2}(v ; \eta, a, \gamma)=\frac{1-\eta}{2} \sum_{j=1}^{n_{T}} s_{\tau_{j}} g\left(v_{\tau_{j}} ; G_{\tau_{j}}, a, \alpha\right), \quad \alpha:=\frac{2 \gamma}{1-\eta},
$$

with $g: \mathbb{R}^{3} \rightarrow \mathbb{R}$ defined in (5.7). Condition (5.10) follows by applying Lemma 5.3 to all terms of the sum in (5.11) and by replacing $\alpha$ given in (5.11) into (5.8).

TheOREM 5.5. Let $\phi(\cdot ; a)$ be the function in (2.1) and $e_{\max } \in \mathbb{R}_{+}^{*}$ the maximum eigenvalue of matrix $B$ in (5.6). Then, if parameters $\lambda, \eta$, a satisfy (1.4) and

$$
a<\bar{a}:=2 \frac{\eta}{1-\eta} \frac{1}{1+\eta e_{\max } / \lambda} \Longleftrightarrow a=\tau_{c} \bar{a}, \tau_{c} \in[0,1),
$$

the function $\mathcal{J}$ in (4.5) is strongly convex over any feasible set defined by a linear constraint of the form in (5.1)-(5.2), hence models in (5.3) admit a unique solution.

Proof. If condition (5.12) holds true, then we can clearly write:

$$
a=\frac{2 \gamma}{1-\eta}, \quad \gamma=\tau_{c} \frac{\eta}{1+\eta e_{\max } / \lambda}, \tau_{c} \in[0,1) .
$$

Based on (5.13), (5.5) and (5.10) and recalling that the sum of a strongly convex and a convex function is strongly convex, the function $\mathcal{J}=\mathcal{J}_{1}+\mathcal{J}_{2}+\mathcal{A}$ in (5.4) is strongly convex over any feasible set defined by a linear constraint of the form in (5.2).

6. Special variants of the model. In this section, we outline some special variants of our decomposition model to be used when some a priori knowledge about the process of formation of the measured field $b=v+w+\epsilon$ is available. In particular, we consider the three cases where, respectively, $v, w$ or $\epsilon$ can be considered as negligible. Starting from (4.5), the special variants read

$$
\begin{aligned}
& v \approx 0 \Longrightarrow v^{*}=0, \quad \epsilon^{*}=b-w^{*}, \quad w^{*} \in \underset{w \in \mathbb{R}^{n_{V}}}{\operatorname{argmin}} \mathcal{J}_{S 1}(w ; \alpha), \quad \alpha=\eta / \lambda, \\
& w \approx 0 \Longrightarrow w^{*}=0, \quad \epsilon^{*}=b-v^{*}, \quad v^{*} \in \underset{v \in \mathbb{R}^{n_{V}}}{\operatorname{argmin}} \mathcal{J}_{S 2}(v ; \alpha, a), \quad \alpha=(1-\eta) / \lambda, \\
& \epsilon \approx 0 \Longrightarrow \epsilon^{*}=0, \quad w^{*}=b-v^{*}, \quad v^{*} \in \underset{v \in \mathbb{R}^{n_{V}}}{\operatorname{argmin}} \mathcal{J}_{S 3}(v ; \alpha, a), \quad \alpha=(1-\eta) / \eta,
\end{aligned}
$$

where the objective functions have the form

$$
\begin{aligned}
\mathcal{J}_{S 1}(w ; \alpha) & =\left\|S_{V}^{1 / 2}(w-b)\right\|_{2}^{2}+\alpha\left\|S^{1 / 2} D w\right\|_{2}^{2}, \\
\mathcal{J}_{S 2}(v ; \alpha, a) & =\left\|S_{V}^{1 / 2}(v-b)\right\|_{2}^{2}+\alpha \sum_{j=1}^{n_{T}} s_{\tau_{j}} \phi\left(\left\|(D v)_{j}\right\|_{2} ; a\right), \\
\mathcal{J}_{S 3}(v ; \alpha, a) & =\left\|S^{1 / 2} D(v-b)\right\|_{2}^{2}+\alpha \sum_{j=1}^{n_{T}} s_{\tau_{j}} \phi\left(\left\|(D v)_{j}\right\|_{2} ; a\right) .
\end{aligned}
$$

We remark that the first two special models above correspond to a pure denoising (no decomposition) by means of an $\ell_{2}$ fidelity term together with a Tikhonov regularization term and a non-convex TV-like regularization term, respectively. The last special model, instead, carries out a pure decomposition (no denoising).

Finally, in the special case where the scalar fields are images, that is the domain is a $2 \mathrm{D}$ rectangle discretized through a regular mesh, then our complete model in 
(4.5) and the three special variants outlined above simplify due to the area of all the mesh elements being the same: matrices $S, S_{V}$ and scalars $s_{\tau_{j}}$ disappear from the models. Moreover, if a standard forward finite difference discretization of the gradient operator is used, then the maximum eigenvalue $e_{\max }$ of matrix $B$ is equal to 8 .

7. Applying ADMM to the proposed CNC model. In this section we describe the ADMM-based algorithm used to solve the proposed constrained model (5.3) in case that parameters $\lambda, \eta, a$ satisfy (1.4) and (5.12), so that the solution is unique. Although such desirable property holds for the infinity of linear constraints defined in (5.2), the constraints might not be equivalent in terms of computational effort implied by the numerical solution. Also, a preliminary change of variables holds the potential for a faster solution. We introduce the one-to-one change of variables

$$
\left\{\begin{array}{l}
u=v+w \\
v=v
\end{array}\right.
$$

and choose the simple constraint

$$
v_{1}=0 \Longleftrightarrow\langle\hat{c},(u ; v)\rangle=0, \quad \hat{c}=\left(0_{n_{V}} ; 1 ; 0_{n_{V}-1}\right) .
$$

It is easy to prove that the transformed-domain constraint (7.2) corresponds to a constraint in the original domain which belongs to the set $\mathcal{C}$ in (5.2). The chosen constraint (7.2) allows also for its direct inclusion into the cost function. In fact, the variable $v$ appears in the cost function only left-multiplied by matrix $D$ and, hence, we can define the reduced vector $\tilde{v} \in \mathbb{R}^{n_{V}-1}$ and matrix $\widetilde{D} \in \mathbb{R}^{3 n_{T} \times\left(n_{V}-1\right)}$ as follows

$$
\tilde{v}:=\left(v_{2}, v_{3}, \ldots, v_{n_{V}}\right)^{T}, \quad \widetilde{D}:=\left(D_{2}, D_{3}, \ldots, D_{n_{V}}\right),
$$

with $D_{i} \in \mathbb{R}^{3 n_{T}}$ denoting the $i$-th column of matrix $D$ in (3.7). Model (5.3) after change of variable (7.1) with constraint (7.2) and after introducing the auxiliary variable $t=\widetilde{D} \tilde{v} \in \mathbb{R}^{3 n_{T}}$, can be reformulated in the equivalent constrained form:

$$
\begin{aligned}
\left\{x^{*}, t^{*}\right\} \leftarrow \arg \min _{x, t}\left\{\frac{\lambda}{2}\left\|S_{V}^{1 / 2}(u-b)\right\|_{2}^{2}+\frac{\eta}{2}\left\|S^{1 / 2}(D u-t)\right\|_{2}^{2}\right. \\
\left.+\frac{(1-\eta)}{2} \sum_{j=1}^{n_{T}} s_{\tau_{j}} \phi\left(\left\|t_{j}\right\|_{2} ; a\right)\right\} \text { subject to }: t=\widetilde{D} \tilde{v}
\end{aligned}
$$

with $x:=(u ; \tilde{v})^{T} \in \mathbb{R}^{2 n_{V}-1}$. To solve (7.4) we define the augmented Lagrangian

$$
\begin{aligned}
\mathcal{L}(x, t, \rho ; \lambda, \eta, a)= & \frac{\lambda}{2}\left\|S_{V}^{1 / 2}(u-b)\right\|_{2}^{2}+\frac{\eta}{2}\left\|S^{1 / 2}(D u-t)\right\|_{2}^{2}+\frac{(1-\eta)}{2} \sum_{j=1}^{n_{T}} s_{\tau_{j}} \phi\left(\left\|t_{j}\right\|_{2} ; a\right) \\
& -\langle\rho, t-\widetilde{D} \tilde{v}\rangle+\frac{\beta}{2}\|t-\widetilde{D} \tilde{v}\|_{2}^{2}
\end{aligned}
$$

with $\beta>0$ a penalty parameter and $\rho \in \mathbb{R}^{3 n_{T}}$ the vector of Lagrange multipliers associated with constraint $t=\widetilde{D} \tilde{v}$ in (7.4). Given the previously computed (or initialized for $k=0$ ) vectors $t^{(k)}$ and $\rho^{(k)}$, the $k$-th iteration of the proposed ADMM-based iterative scheme for the computation of the saddle-point of $\mathcal{L}$ in (7.5) reads as follows:

$$
\begin{aligned}
& x^{(k+1)} \leftarrow \underset{x \in \mathbb{R}^{2 n_{V}-1}}{\operatorname{argmin}} \mathcal{L}\left(x, t^{(k)}, \rho^{(k)} ; \lambda, \eta, a\right), \\
& t^{(k+1)} \leftarrow \underset{t \in \mathbb{R}^{3 n_{T}}}{\operatorname{argmin}} \mathcal{L}\left(x^{(k+1)}, t, \rho^{(k)} ; \lambda, \eta, a\right), \\
& \rho^{(k+1)} \leftarrow \rho^{(k)}-\beta\left(t^{(k+1)}-\widetilde{D} \tilde{v}^{(k+1)}\right) .
\end{aligned}
$$


The first-order optimality conditions for the quadratic sub-problem for $x$ in (7.6) read

$$
\left(\begin{array}{cc}
S_{V}+(\eta / \lambda) D^{T} S D & 0 \\
0 & \widetilde{D}^{T} \widetilde{D}
\end{array}\right)\left(\begin{array}{l}
u \\
\tilde{v}
\end{array}\right)=\left(\begin{array}{l}
S_{V} b+(\eta / \lambda) D^{T} S t^{(k)} \\
\widetilde{D}^{T}\left(t^{(k)}-\frac{1}{\beta} \rho^{(k)}\right)
\end{array}\right),
$$

with $\widetilde{D} T \widetilde{D} \in \mathbb{R}^{\left(n_{V}-1\right) \times\left(n_{V}-1\right)}$ a full rank matrix. We remark that system (7.9) is decoupled in $u$ and $\tilde{v}$ thanks to the chosen preliminary change of variables.

After some algebraic manipulations, and introducing the constant (with respect to the optimization variable $t$ ) vectors $r_{j}^{(k+1)} \in \mathbb{R}^{3}, j=1, \ldots, n_{T}$, defined by

$$
r_{j}^{(k+1)}=\frac{1}{\eta s_{\tau_{j}}+\beta}\left(s_{\tau_{j}} \eta\left(D u^{(k+1)}\right)_{j}+\beta\left(\widetilde{D} \tilde{v}^{(k+1)}+\frac{1}{\beta} \rho^{(k)}\right)_{j}\right),
$$

the sub-problem for $t$ in (7.7) can be decoupled into the $n_{T}$ element-wise problems

$$
t_{j}^{(k+1)} \leftarrow \arg \min _{t \in \mathbb{R}^{3}}\left\{\phi\left(\left\|t_{j}\right\|_{2} ; a\right)+\frac{\omega_{j}}{2}\left\|t_{j}-r_{j}^{(k+1)}\right\|_{2}^{2}\right\}, \quad \omega_{j}=2 \frac{\eta+\beta / s_{\tau_{j}}}{1-\eta} .
$$

Since we are assuming that condition (5.12) is satisfied, so that our cost function is strongly convex, we aim at avoiding non-convexity of the ADMM sub-problems (7.11). In Proposition 7.1 below, we give necessary and sufficient conditions for strong convexity of the cost functions in (7.11). In particular, based on (7.14)-(7.15), the problems in (7.11) are strongly convex if and only if the following condition holds:

$$
\beta>(a(1-\eta) / 2-\eta) \max _{j} s_{\tau_{j}} .
$$

In case that (7.12) is fulfilled, the unique solutions of problems in (7.11) can be obtained based on the operator defined in (7.17)-(7.18) of Proposition 7.1, that is:

$$
t_{j}^{(k+1)}=\xi_{j}^{(k+1)} r_{j}^{(k+1)}=\min \left\{\max \left\{\nu_{j}-\zeta_{j} /\left\|r_{j}^{(k+1)}\right\|_{2}, 0\right\}, 1\right\} r_{j}^{(k+1)},
$$

where $\nu_{j}=\omega_{j} /\left(\omega_{j}-a\right), \zeta_{j}=\sqrt{2 a} /\left(\omega_{j}-a\right)$, with $\omega_{j}$ defined in (7.11).

Proposition 7.1. Let $\phi(\cdot ; a)$ be the penalty function defined in (2.1), $r \in \mathbb{R}^{m}$ a given constant vector and $a, \omega \in \mathbb{R}_{+}^{*}$ two (free) parameters. Then, the function

$$
\theta(z):=\phi\left(\|z\|_{2} ; a\right)+\frac{\omega}{2}\|z-r\|_{2}^{2}, \quad z \in \mathbb{R}^{m},
$$

is strongly convex if and only if the following condition is satisfied:

$$
\omega>a .
$$

Moreover, in case that (7.15) holds, the strongly convex minimization problem

$$
\arg \min _{z \in \mathbb{R}^{m}} \theta(z)
$$

admits the unique solution $z^{*} \in \mathbb{R}^{m}$ given by the following shrinkage operator:

$$
\begin{gathered}
z^{*}=\xi^{*} r, \quad \text { with } \xi^{*} \in[0,1], \text { in particular : } \\
\xi^{*}=\min \left\{\max \left\{\nu-\zeta /\|r\|_{2}, 0\right\}, 1\right\}, \quad \nu=\omega /(\omega-a), \quad \zeta=\sqrt{2 a} /(\omega-a) .
\end{gathered}
$$

The proof is postponed to the Appendix.

We remark that the condition on $\beta$ in (7.12) only ensures (strong) convexity of the sub-problems for the primal variable $t$, but does not guarantee convergence of the overall ADMM scheme. Following [23], a proof of convergence of the proposed ADMM-based minimization algorithm will be investigated in future work. 

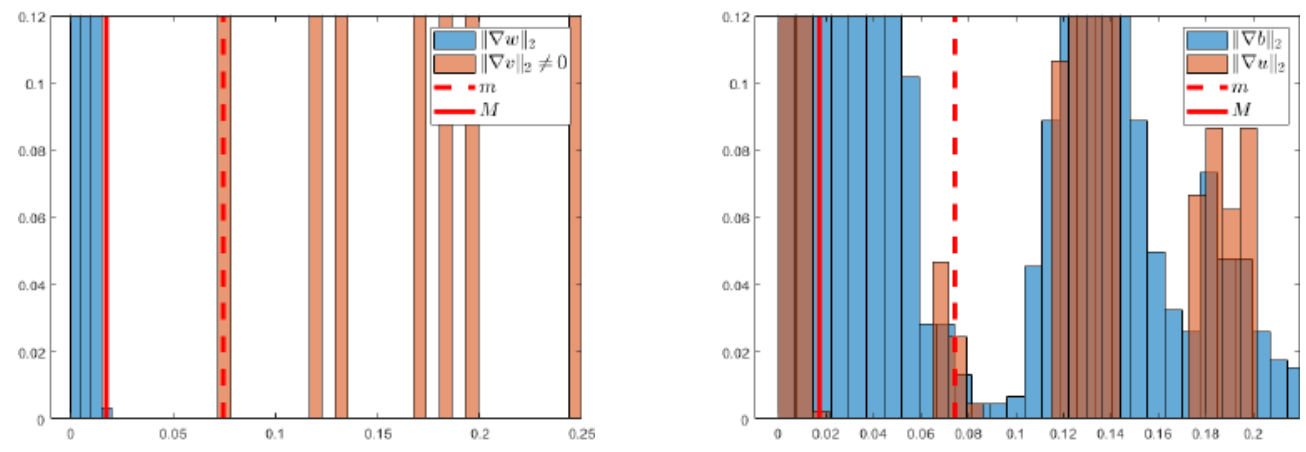

FIG. 8.1. Left: normalized histograms of the gradient magnitudes of components $v$ and $w$ reported in Fig. 1.1. Right: normalized histograms of the gradient magnitudes of the noiseless image $u=v+w$ and of the noise-corrupted image $b=v+w+\epsilon$.

8. Parameters selection. In this section we discuss how the free parameters $a, \lambda, \eta$ in our decomposition model (4.5), (5.3) can be suitably set.

First, for what regards $a$, in $\mathrm{CNC}$ regime we suggest to set $\tau_{c}$ in (5.12) strictly less than but near to 1 . Clearly, one can use our decomposition model also in pure non-convex regime by setting $\tau_{c}$ greater than 1 or in pure convex regime by letting $\tau_{c}$ tend to zero, such that the regularizer $\mathcal{R}_{v}$ in our cost functional (4.5) tends to the classical convex TV regularizer. We also notice that by letting $\tau_{c}$ tend to $+\infty$ then the penalty $\phi$ in (2.1) tends to the $\ell_{0}$ pseudo-norm, hence sparsity of the gradient norms of component $v$ is maximally promoted by regularizer $\mathcal{R}_{v}$.

For what concerns $\lambda$, we rely on the discrepancy principle which, given a known - or estimated - value for the noise standard deviation $\hat{\sigma}$, suggests to choose a $\lambda$ such that the obtained solution $u^{*}=v^{*}+w^{*}$ satisfies

$$
\left\|u^{*}-b\right\|_{2} \leq \tau_{d} \sqrt{n_{V}} \hat{\sigma},
$$

with $\tau_{d}$ a coefficient usually chosen around 1 .

For what regards $\eta$, we present a procedure for automatically setting its value under the assumption that we can get good estimates of the minimum non-zero gradient norm of the cartoon component $v$ - that is, the minimum size of jump discontinuities in $v$ - and of the maximum gradient norm of the smooth component $w$, namely of

$$
m:=\min _{j:\left\|(\nabla v)_{j}\right\|_{2}>0}\left\|(\nabla v)_{j}\right\|_{2}, \quad M:=\max _{j}\left\|(\nabla w)_{j}\right\|_{2}, \quad j=1,2, \ldots, n_{T} .
$$

In real applications, clearly one does not know $v$ and $w$ in advance. However, we can estimate $m$ and $M$ directly from the observed scalar field $b$ and then proceed as described below to estimate the $\eta$ parameter value. In Fig. 8.1(left) we show zooms of the normalized histograms of gradient norms of components $v$ and $w$ shown in the first row of Fig. 1.1, together with two vertical lines representing $m$ and $M$ defined in (8.2). It is clear that $M<m$, meaning that the histograms for $v$ and $w$ are separable and that, likely, $M$ and $m$ can be robustly estimated starting from the histogram of the gradient norms of the noise-free observation $u=v+w$, shown in Fig. 8.1(right). The second histogram in Fig. 8.1(right) is the one associated to the noisy observation $b=u+\epsilon$, with $\epsilon$ the realization of white Gaussian noise of standard deviation $\sigma=2 / 255$. Automatic estimation of $m$ and $M$ also in case of noisy observations is a more challenging task, and will be a point of future investigation. 
We note that - see $(4.5)$ - parameter $\eta \in(0,1)$ controls the convex combination of the two regularizers for components $v$ and $w$, which can be usefully rewritten as

$$
\frac{1-\eta}{2} \mathcal{R}_{v}(v ; a)+\frac{\eta}{2} \mathcal{R}_{w}(w)=\frac{1}{2} \sum_{j=1}^{n_{T}} s_{\tau_{j}}\left[f_{v}\left(\left\|(D v)_{j}\right\|_{2} ; a, \eta\right)+f_{w}\left(\left\|(D w)_{j}\right\|_{2} ; \eta\right)\right]
$$

where we introduced the functions $f_{v}, f_{w}: \mathbb{R}_{+} \rightarrow \mathbb{R}_{+}$defined by

$$
f_{v}(t ; a, \eta):=(1-\eta) \phi(t ; a), \quad f_{w}(t ; \eta):=\eta t^{2}, \quad t \in \mathbb{R}_{+} .
$$

Our proposal is to set $\eta$ such that $f_{v}, f_{w}$ above intersect at a point of abscissa

$$
\bar{t}=\kappa:=(m+M) / 2 .
$$

In Proposition 8.1 below, whose proof is given in the Appendix, we provide a formula that, for any fixed value of parameters $a, \lambda$, allows to compute the unique $\eta$ yielding the prescribed abscissa $\bar{t}$ of the intersection between functions $f_{v}$ and $f_{w}$ in (8.3).

The idea at the basis of the proposed strategy is that, even though the decomposition process carried out by the proposed variational model is global to the whole fields domain, by setting $\eta$ as above we expect to likely push locally large/small gradients - i.e., edges/smooth parts - into the estimated cartoon/smooth component $v^{*} / w^{*}$.

Proposition 8.1. Let $\phi$ be the penalty function defined in (2.1) and let parameters $\lambda, \eta$, a satisfy (1.4). Then, the functions $f_{v}(t ; a, \eta)$ and $f_{w}(t ; \eta)$ defined in (8.3) intersect at the origin and at another unique point having abscissa $\bar{t}>0$ defined by

$$
\begin{gathered}
\bar{t}= \begin{cases}\sqrt{\frac{1-\eta}{\eta}} & \text { if } \eta \in\left(0, \eta_{12}\right] \Longleftrightarrow \tau_{c} \in\left[\tau_{c 12}^{\prime}, \infty\right), \\
\sqrt{\frac{1-\eta}{\eta} \frac{2 \sqrt{C}}{1+C}} & \text { otherwise }\end{cases} \\
C=\tau_{c} /(1+\eta \nu), \quad \eta_{12}=\left(\tau_{c}-1\right) / \nu, \quad \tau_{c 12}^{\prime}=1+\eta \nu, \quad \nu=e_{\max } / \lambda .
\end{gathered}
$$

Then, for any given $\left(\lambda, \tau_{c}\right),(8.5)$ can be inverted to get $\eta$ as a function of $\bar{t}$, namely:

$$
\begin{gathered}
\eta=\left\{\begin{array}{l}
\frac{1}{1+\bar{t}^{2}} \quad \text { if } \bar{t} \in\left(0, \bar{t}_{12}\right] \Longleftrightarrow \tau_{c} \in\left[\tau_{c 12}^{\prime \prime}, \infty\right) \\
\text { unique solution in }(0,1) \text { of } P_{0}+P_{1} \eta+P_{2} \eta^{2}+P_{3} \eta^{3}=0 \text { otherwise }
\end{array}\right. \\
P_{0}=-4 \tau_{c}, P_{1}=\left(1+\tau_{c}\right)^{2} \bar{t}^{2}+4 \tau_{c}(1-\nu), P_{2}=2 \nu\left(\left(1+\tau_{c}\right) \bar{t}^{2}+2 \tau_{c}\right), P_{3}=\bar{t}^{2} \nu^{2}, \\
\bar{t}_{12}=\sqrt{\nu /\left(\tau_{c}-1\right)-1}, \quad \tau_{c 12}^{\prime \prime}=1+\nu /\left(1+\bar{t}^{2}\right) .
\end{gathered}
$$

8.1. Validating the $\eta$ parameter selection strategy.. In order to better explain as well as to validate the idea at the basis of the proposed parameter selection strategy, we now carry out a numerical experiment consisting in applying our CNC model to the decomposition of image $u$ shown in the first row of Fig. 1.1, both in the noiseless and in the noisy case. To quantitatively evaluate the quality of the obtained decomposition results, we use the Signal-to-Noise Ratio (SNR) defined by $\operatorname{SNR}\left(x^{*}, x\right):=10 \log _{10}\left(\|x-E[x]\|_{2}^{2} /\left\|x^{*}-x\right\|_{2}^{2}\right)$, with $x^{*}$ the computed estimate of the original scalar field $x$ and $E[x]$ denoting the mean value of $x$.

Noise-free images. In the first row of Fig. 8.2 we show the SNR plots for the estimated components $v^{*}$ and $w^{*}$ obtained by decomposing the noise-free image $u$ in the first 
row of Fig. 1.1 by using the third (S3) special decomposition model outlined in Sect. 6 with parameters $\tau_{c}=0.99$ and $\eta$ varying in its domain $(0,1)$. In particular, we limit the plots to the sub-domain of $\eta$ yielding non-zero SNR values. The red circles in the plots correspond to the values $\eta \in\{0.78,0.9,0.996,0.99999\}$. The decompositions obtained by using such $\eta$ values are shown in the second and third row of Fig. 8.2.

According to the results demonstrated in Proposition 8.1 - in particular, formula (8.7) - the $\eta$ value yielding the abscissa of the intersection point between $f_{v}$ and $f_{w}$ to be $\bar{t}=\kappa=(m+M) / 2$ in this experiment is $\eta=0.9978$. By observing the SNR graphs in the first row of Fig. 8.2, one can notice that the maximum achieved SNR values are in proximity of that $\eta$ value. Moreover, by observing the plots reported in the last row of Fig. 8.2 one can notice that the best decomposition - see the case c) - is obtained in correspondence of an $\eta$ value yielding the abscissa of the intersection point between the functions $f_{v}$ and $f_{w}$ defined in (8.3) to be located inside the interval $[M, m]$. This means that the gradient norms which are lower than such abscissa are penalized more strongly by $f_{v}$, vice-versa for gradient norms which are larger.

Noisy images. We now apply our complete model in (4.5), (5.3) with parameter $\tau_{c}=0.99$ to decomposing the noisy image $b=u+\epsilon$ with $u=v+w$, again, the noisefree image in the first row of Fig. 1.1 and $\epsilon$ the realization of white Gaussian noise of standard deviation $\sigma=5 / 255$. In this case, for any fixed $\tau_{c}$ the value of parameter $\eta$ provided by the formula in (8.7) depends on $\bar{t}$ and also on $\lambda$, as illustrated in Fig. 8.3(a) for $\lambda \in[0.1,20]$. The size of the interval $[\eta(\bar{t}=M, \lambda), \eta(\bar{t}=m, \lambda)]$ of most likely good values for $\eta$ changes significantly with $\lambda$. In order to focus the analysis of the performance of our model over choices of $\bar{t}$ - hence of the associated $\eta$ - in the vicinity of the suggested value $\bar{t}=\kappa=(m+M) / 2$, we propose the following $\kappa$-centered affine re-parametrization of $\bar{t}$ with new parameter $\delta$ :

$$
\bar{t}(\delta ; m, M)=\frac{m+M}{2}+\frac{|m-M|}{2} \delta=\kappa+\frac{|m-M|}{2} \delta,
$$

illustrated in Fig. 8.3(b) for $\delta \in[-1.2,1.2]$.

In Fig. 8.4 we show the SNR surfaces obtained by running our model with parameters $\tau_{c}=0.99$ and $(\lambda, \eta(\bar{t}(\delta)))$ varying in the domain $[0.1,20] \times[-1.2,1.2]$, where $\bar{t}(\delta ; m, M)$ is given in (8.9) and $\eta(\bar{t})$ in (8.7)-(8.8). In Fig. 8.4 we also plot three lines corresponding to the values $\delta \in\{-1,0,1\}$ yielding $\bar{t} \in\{M, \kappa, m\}$, respectively, and in the other direction three lines representing the iso-curves of the discrepancy equal to $\{3,4,5\}$. We can see that the maximum $\operatorname{SNR}\left(u^{*}\right)$ is obtained for the discrepancy isocurve level 4 , marked by $\bullet$, reaching the $\operatorname{SNR}\left(u^{\bullet}\right)=32.56$, while $\star$ marks the point of maximum $\mathrm{SNR}_{\text {mean }}$ given by the average of $\operatorname{SNR}\left(u^{*}\right), \operatorname{SNR}\left(v^{*}\right)$ and $\operatorname{SNR}\left(w^{*}\right)$. The corresponding images output of the decomposition process are reported in Fig. 8.5. We observe from Figs. 8.4, 8.5 that the proposed parameters-setting strategy allows to robustly achieve good quality results.

Different blendings. We finally validate the $\eta$-setting strategy in (8.4) for different blendings of the two components $v$ and $w$ shown in Fig. (1.1), yielding different values of the $(m, M)$ pair in (8.2) - hence, of the $\kappa$ value in (8.4). In particular, we apply our CNC model $\left(\tau_{c}=0.99\right)$ to different linear mixtures of $v$ and $w$, namely:

$$
b(z):=2(1-z) v+2 z w, \quad z \in(0,1) .
$$

In Fig. 8.6 we show the estimated $v^{*}$ and $w^{*}$ components and, in the captions, the associated SNR values for the three cases $z \in\{0.2,0.5,0.8\}$ in (8.10). These results 

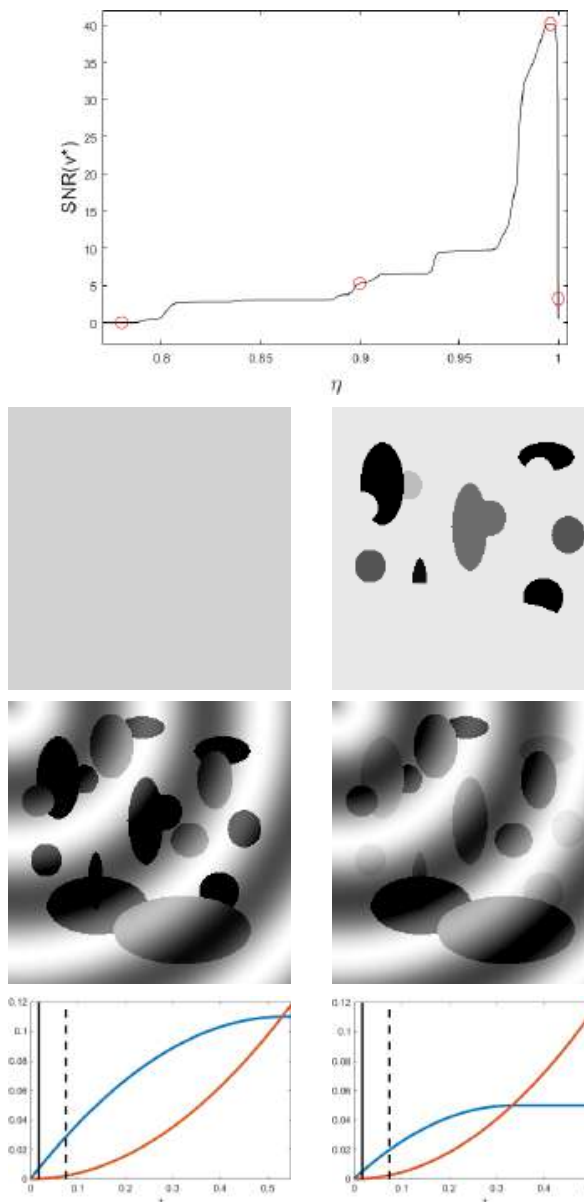

a) $\eta=0.78$

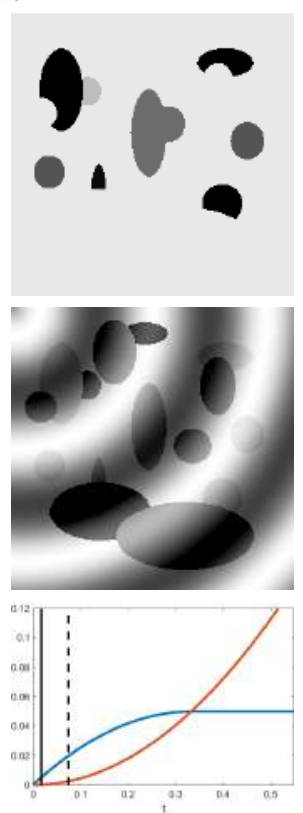

b) $\eta=0.9$
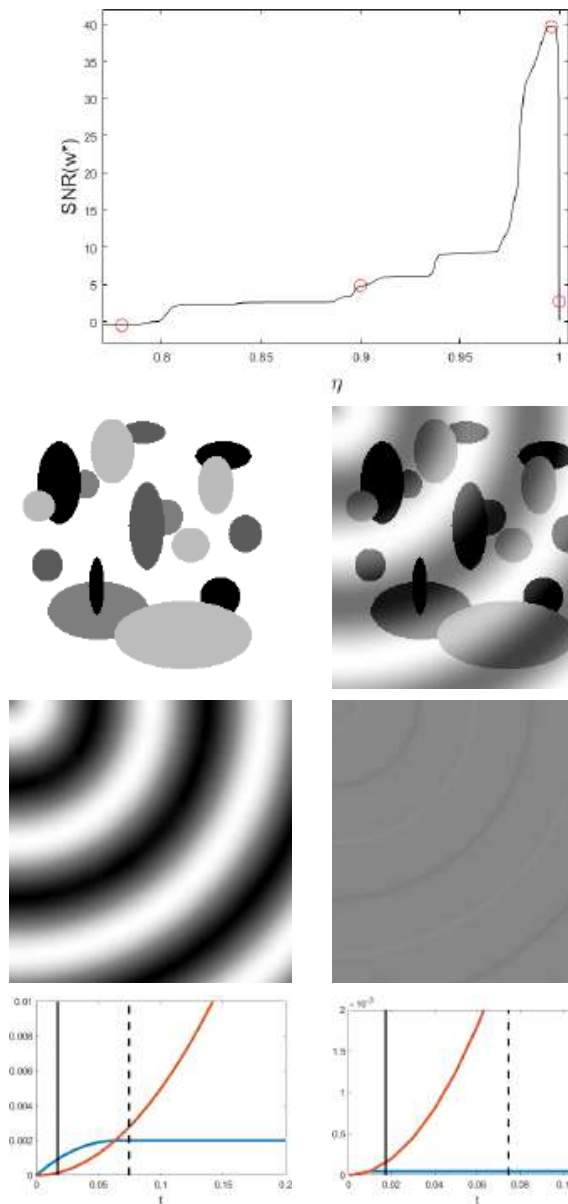

c) $\eta=0.996$ $\eta$

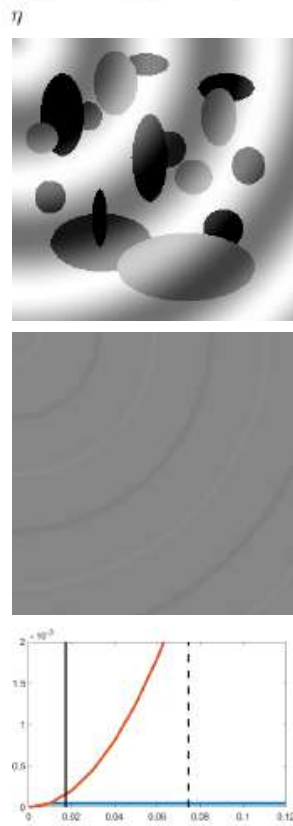

d) $\eta=0.9999$

FIG. 8.2. SNR plots for varying $\eta$ parameter values (first row), resulting $v^{*}$ (second row) and $w^{*}$ (third row) for $\eta$ values corresponding to the red circles in the graphs of the first row. Corresponding plots of the $f_{v}(t ; \eta, a)$ (blue) and $f_{w}(t ; \eta)$ (red) functions defined in (8.3) (fourth row). $M$ is marked by a solid line, while $m$ by a dashed line.

confirm that, as far as the gradient norms of $v$ and $w$ are well separable, our CNC model coupled with the $\eta$-setting strategy achieves good results.

9. Numerical Experiments. In this section, we evaluate experimentally the performance of the proposed variational decomposition approach when applied to images (Example 1) and scalar fields defined on surfaces (Examples 2 and 3).

For all the experiments, the parameters $m$ and $M$ are estimated from noiseless scalar fields and also used for the decomposition of the noisy scalar fields. For all the tests, the iterations of the ADMM algorithm in (7.6)-(7.8) are stopped as soon as either of the two following conditions is fulfilled:

$$
k>\mathrm{TH}_{1}=1000, \quad\left\|x^{(k+1)}-x^{(k)}\right\|_{2} /\left\|x^{(k)}\right\|_{2}<\mathrm{TH}_{2}=10^{-6} .
$$




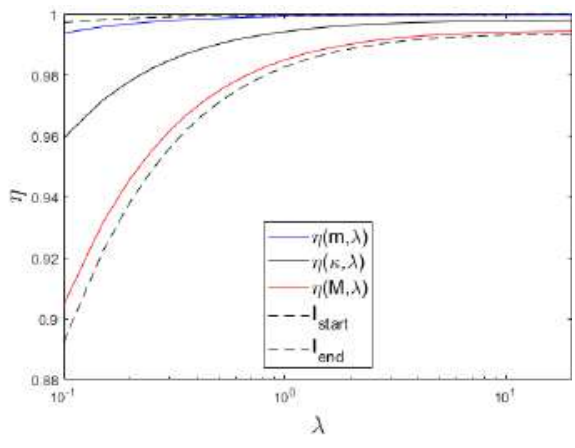

a) $(\lambda, \eta(\bar{t}, \lambda))$

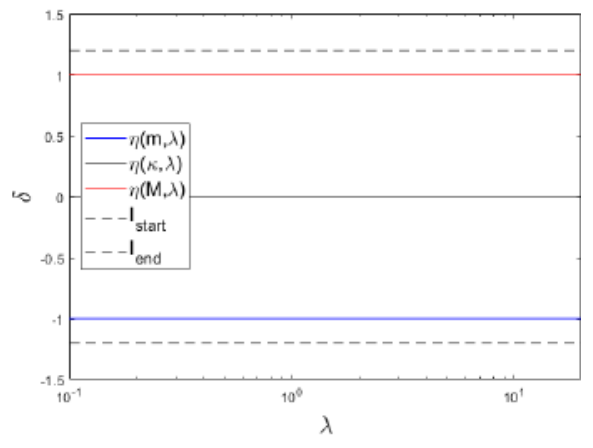

b) $(\lambda, \delta(\lambda))$

FIG. 8.3. (a) Plot of $\eta$ values in terms of $\lambda$; (b) reparametrization in $\delta$.

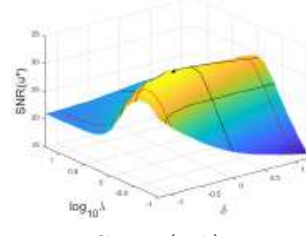

$\operatorname{SNR}\left(u^{*}\right)$

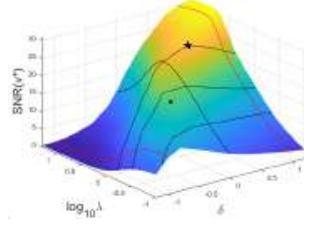

$\operatorname{SNR}\left(v^{*}\right)$

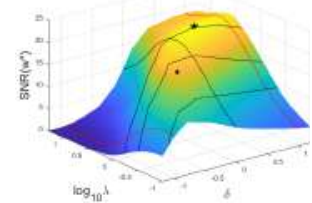

$\operatorname{SNR}\left(w^{*}\right)$

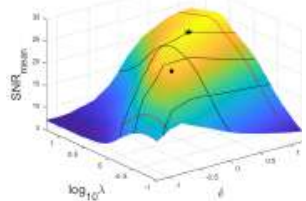

$\mathrm{SNR}_{\text {mean }}$

FIG. 8.4. SNR surfaces for $u^{*}, v^{*}, w^{*}$ and their mean.

9.1. Example 1. In this first example, we investigate the performance of the proposed variational model when applied to the decomposition of images, both in case of noise-free and in case of noise-corrupted observations $b$. In particular, in order to provide evidence for usefulness of the CNC strategy, we are interested in testing the performance of our model when applied in its three main working regimes, depending on parameter $\tau_{c}$ in (5.12), namely the purely convex (TV) regime $\left(\tau_{c}=0\right)$, the CNC regime $\left(0<\tau_{c} \leq 1\right)$ and the purely non-convex $(\mathrm{NC})$ regime $\left(\tau_{c}>1\right)$.

\section{Decomposition of noise-free images.}

The synthetic noise-free image $u=v+w$ shown in the first column of Fig. 9.3 has been processed using the decomposition model $\mathcal{J}_{S 3}$ outlined in Sect. 6 with varying values for $\eta(\delta, \cdot)$ obtained by finely sampling the interval $\delta \in[-1,1.5]$. In particular, we run the ADMM algorithm for the $\mathrm{CNC}\left(\tau_{c}=0.99\right), \mathrm{NC}\left(\tau_{c}=5\right)$, and $\mathrm{TV}\left(\tau_{c}=0\right)$ regimes. In Fig. 9.1 we plot $\operatorname{SNR}\left(v^{*}\right)$ and $\operatorname{SNR}\left(w^{*}\right)$ as functions of $\delta$.

First, we observe from the graphs in Fig. 9.1 that our model in the CNC and $\mathrm{NC}$ regimes performs much better than in the TV regime, both in terms of maximum achieved SNR values and in terms of SNR peak shapes and positions. In fact, the SNR curves for $\mathrm{CNC}$ and $\mathrm{NC}$ are almost flat and equal to their maximum in the whole interval $\delta \in[-1,1]$, this validating the proposed $\eta$-setting strategy in these two regimes. Instead, the SNR graphs for TV exhibit a quite narrow peak in correspondence of a low $\delta$ value, thus making the $\eta$-setting procedure very fragile in this regime.

Then, it is very interesting to note that CNC and NC performs very similarly, with the crucial difference that in the CNC case our model is strongly convex - with all the well-known associated good properties - whereas non-convexity of the model in the NC case can well be source of intrinsic (existence of local minimizers) as well as numerical (convergence of the minimization algorithm) problems. In fact, by comparing the 


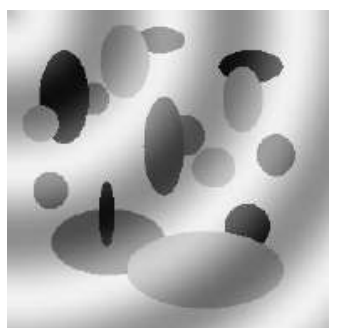

$u$

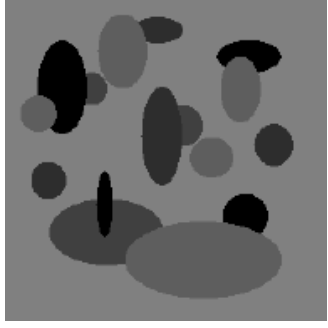

$v$

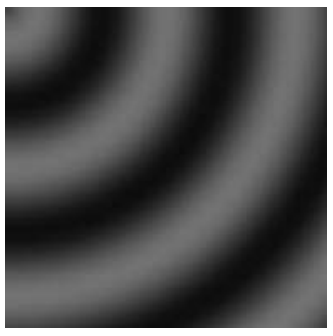

$w$

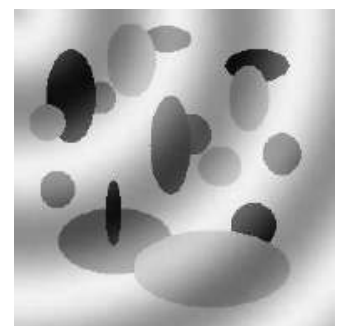

$\operatorname{SNR}\left(u^{\bullet}\right)=32.55$

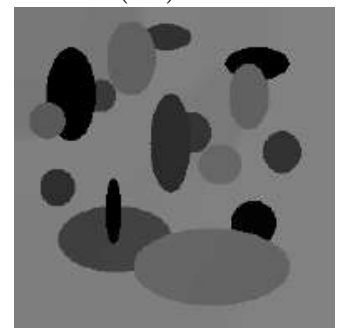

$\operatorname{SNR}\left(v^{\bullet}\right)=18.88$

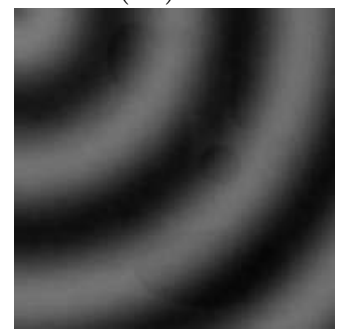

$\operatorname{SNR}\left(w^{\bullet}\right)=18.25$

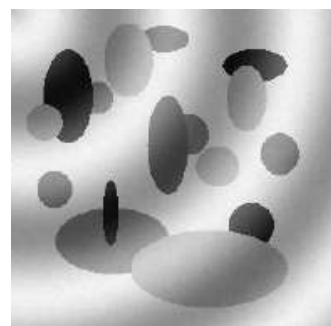

$\operatorname{SNR}\left(u^{\star}\right)=27.84$

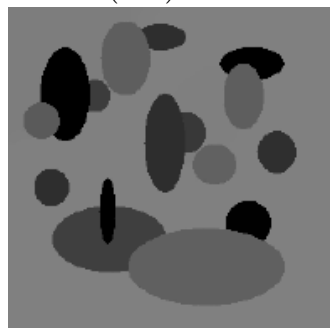

$\operatorname{SNR}\left(v^{\star}\right)=27.03$

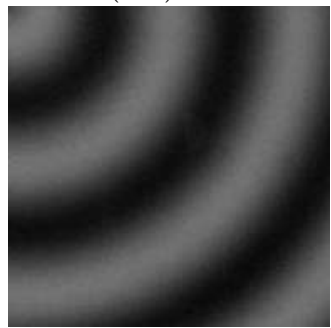

$\operatorname{SNR}\left(w^{\star}\right)=22.23$

FIG. 8.5. Decomposition results obtained from corrupted image with $\mathrm{SNR}(b)=20.99$ in comparison to the original images in the first column. Second column corresponds to the best denoising of $u^{*}$, marked as solid dot in Fig. 8.4. Third column corresponds to maximum SNR of the mean, marked by a star in Fig. 8.4.

$\mathrm{NC}$ and $\mathrm{NC}_{\mathrm{GT}}$ graphs in Fig.9.1, which have been obtained by using a random and a ground-truth initial ADMM iterate, respectively, with stopping criteria in (9.1) defined by $\mathrm{TH}_{1}=+\infty, \mathrm{TH}_{2}=10^{-8}$, one can deduce that $\mathrm{NC}$ with random guess got regularly stuck in local minima with lower SNR values for $\delta>0.75$. These results strongly indicate motivations for using our decomposition model in the CNC regime. Decomposition of noisy images.

We apply our model in the $\mathrm{TV}, \mathrm{CNC}, \mathrm{NC}$ regimes to decomposing the noisy image $b=u+\epsilon$, with $u$ the noiseless image in Fig. 9.3 and $\epsilon$ the realization of white Gaussian noise with standard deviation $\sigma=5 / 255$, yielding to $\operatorname{SNR}(b)=21.33$.

In the first row of Fig. 9.2, we report the $\mathrm{SNR}_{\text {mean }}$ graphs for the three regimes over the parameters domain $(\lambda, \delta) \in[0.1,20] \times[-1,1.5]$. We over-imposed the iso-levels $\{3,4,5\}$ of the discrepancy as well as of $\delta=\{-1,0,1\}$, yielding $\{\eta(M), \eta(\kappa), \eta(m)\}$. $\mathrm{By} \star$ we denote the point of maximum $\mathrm{SNR}_{\text {mean }}$. The corresponding resulting images $u^{\star}, v^{\star}, w^{\star}$ are reported in Fig. 9.3 for the three regimes, together with the original images (first column). We observe from these results that TV is the worst performing especially in terms of $\{u, v\}$ decomposition, whereas CNC and NC perform similarly well, with NC providing slightly better results. 


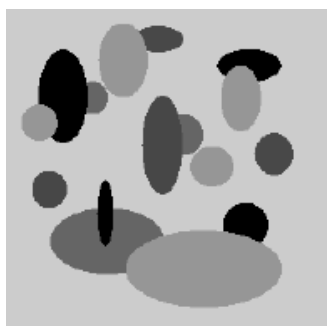

$\operatorname{SNR}\left(v^{*}\right)=52.28$

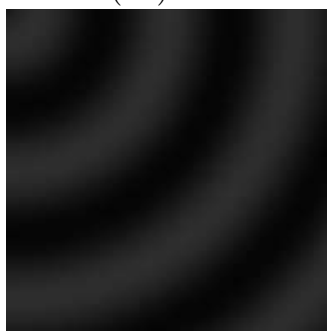

$\operatorname{SNR}\left(w^{*}\right)=39.76$

a) $z=0.2$

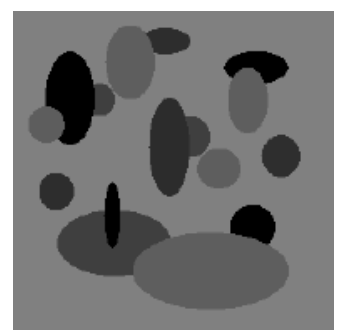

$\operatorname{SNR}\left(v^{*}\right)=40.23$

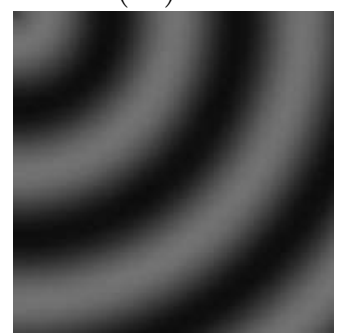

$\operatorname{SNR}\left(w^{*}\right)=39.76$

b) $z=0.5$

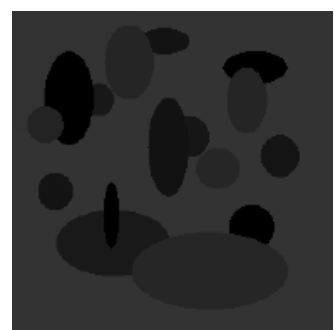

$\operatorname{SNR}\left(v^{*}\right)=20.60$

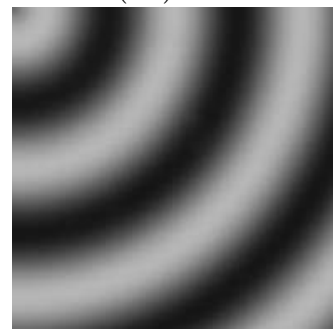

$\operatorname{SNR}\left(w^{*}\right)=32.18$

c) $z=0.8$

FIG. 8.6. Blending: components $v^{*}$ (first row) and $w^{*}$ (second row) estimated by using our $C N C$ decomposition approach $\left(\tau_{c}=0.99\right)$ for three different linear blendings according to (8.10).
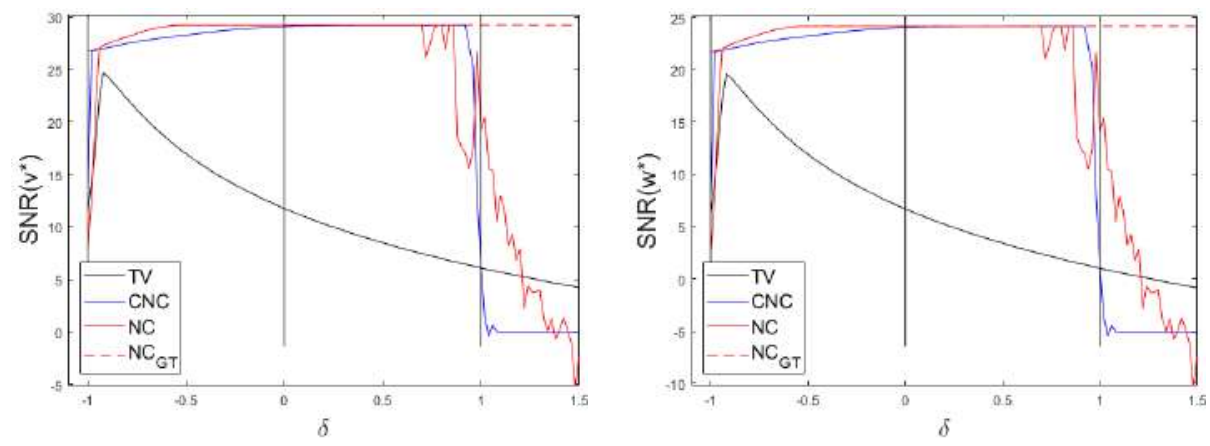

FIG. 9.1. Example 1 (noise-free images): SNR graphs for the decomposition of the noisy-free image $u$ in the first column of Fig. 9.3 into $v^{*}$ and $w^{*}$ components under different regimes.

In the second row of Fig.9.2 we present an empirical investigation on the numerical convergence of the proposed ADMM-based minimization scheme, in the different regimes. We report convergence plots for three different values of the penalty parameter $\beta$ chosen in the interval $\beta \in[0.75,50]$, with a random ADMM starting iterate. The empirical behavior suggests numerical convergence of the convex TV and the proposed $\mathrm{CNC}$ schemes, while in the $\mathrm{NC}$ regime the convergence plots are in general highly oscillating and the method does not converge for $\beta=0.75$. Moreover, as well known and previously pointed out, the NC regime is also dependent on the initialization, which can cause stalling at various different local minima solutions.

9.2. Example 2. In this example we test the proposed decomposition approach in the general case of scalar fields defined over surfaces. In particular, we apply our model in its $\mathrm{CNC}\left(\tau_{c}=0.99\right)$ and, for comparison, $\mathrm{TV}\left(\tau_{c}=0\right)$ modalities to 

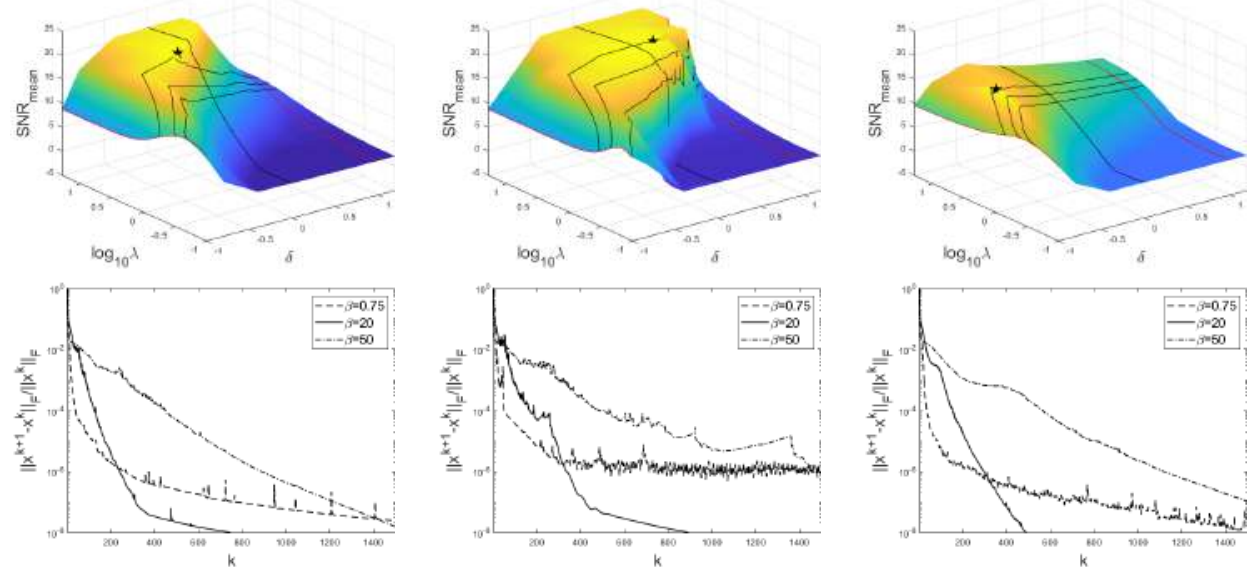

a) $\mathrm{CNC}\left(\tau_{c}=0.99\right)$

b) $\mathrm{NC}\left(\tau_{c}=10\right)$

c) $\operatorname{TV}\left(\tau_{c}=0\right)$

FIG. 9.2. Example 1 (noisy images): $S N R_{\text {mean }}$ graphs for varying $\lambda$ and $\eta$ parameters for the three different regimes (first row). Corresponding ADMM convergence plots (second row).

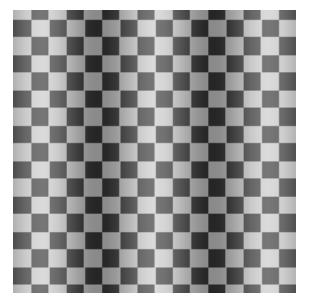

$u$

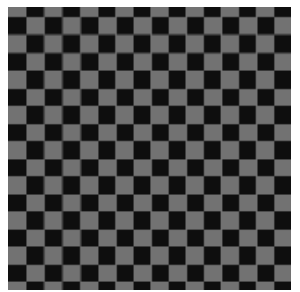

$v$

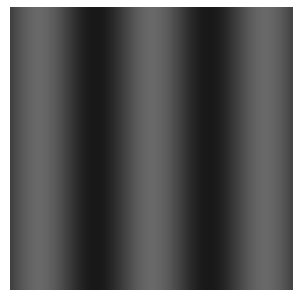

$w$

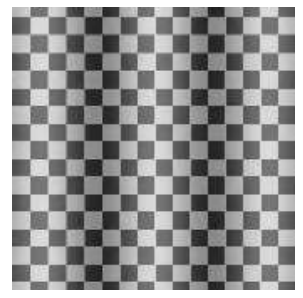

$\operatorname{SNR}\left(u^{\star}\right)=27.41$

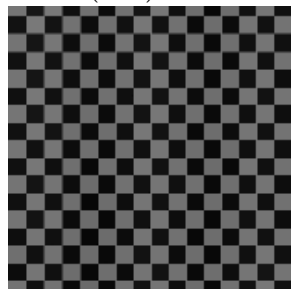

$\operatorname{SNR}\left(v^{\star}\right)=22.97$

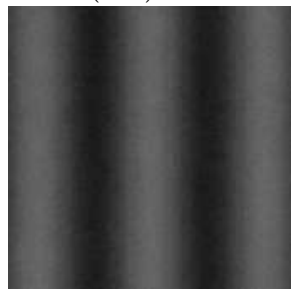

$\operatorname{SNR}\left(w^{\star}\right)=16.39$

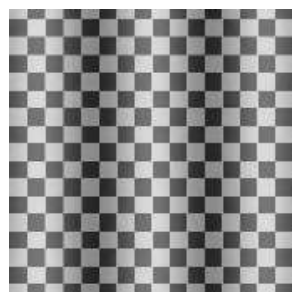

$\operatorname{SNR}\left(u^{\star}\right)=28.42$

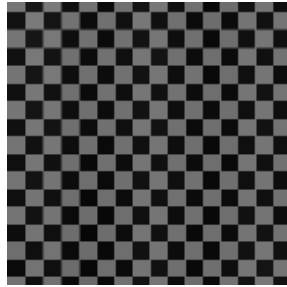

$\operatorname{SNR}\left(v^{\star}\right)=23.35$

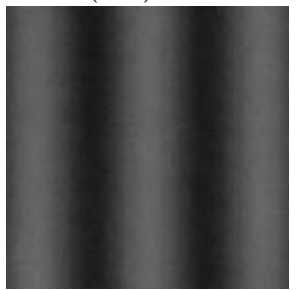

$\operatorname{SNR}\left(w^{\star}\right)=16.99$

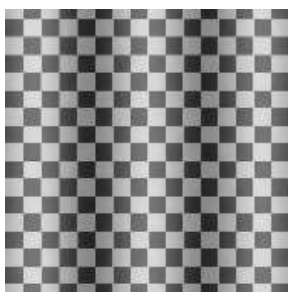

$\operatorname{SNR}\left(u^{\star}\right)=26.18$

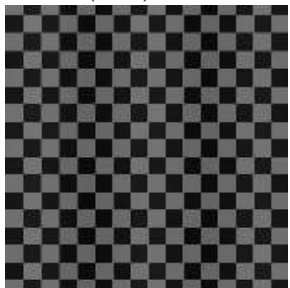

$\operatorname{SNR}\left(v^{\star}\right)=16.13$

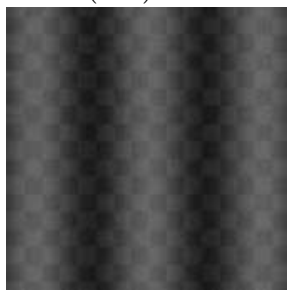

$\operatorname{SNR}\left(w^{\star}\right)=11.52$

FIG. 9.3. Example 1 (noisy images): decompositions of noisy images obtained for the parameters providing highest mean SNR for each regime. Original (first column), CNC (second column), NC (third column) and TV (last column).

decomposing the noiseless piecewise smooth field $u$ in Fig. 9.4, given by the sum of a 


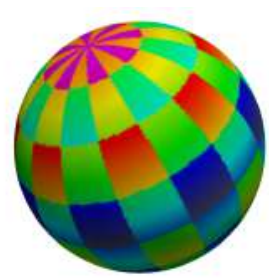

$u$

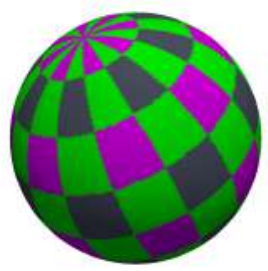

$v$

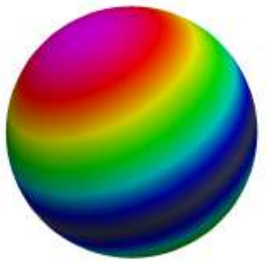

$w$

FIG. 9.4. Example 2: (left) scalar field on a sphere; (middle) piecewise-constant component; (right) piecewise smooth component.

chessboard cartoon field $v$ and a smooth field $w$, both defined over a meshed sphere.

We are especially interested in investigating the impact of the mesh scale, resolution and quality on the obtained decomposition results. To this aim, different sphere meshes $S_{i}=\left\{V_{i}, T_{i}\right\}, i=1, \ldots, 4$, of increasing resolutions $\left|S_{i}\right|:=\left(\left|V_{i}\right|,\left|T_{i}\right|\right)$ were created, namely $\left|S_{1}\right|=(4098,8192),\left|S_{2}\right|=(16386,32768),\left|S_{3}\right|=(65538,131072)$, $\left|S_{4}\right|=(66114,132288)$. Visual details of the meshes are shown in Fig. 9.5, whereas in Table 9.1 we report some quantities characterizing or depending on the meshes, namely the radius $r$ of the meshed sphere, the minimum height $h_{\min }$ of mesh triangles, the maximum eigenvalue $e_{\max }(B)$ of matrix $B$ in (5.6) and the resulting - according to (5.12) - concavity parameter $a$ for our CNC model. We also report some quantities characterizing the scalar fields defined over the meshes, namely $m$ and $M$ defined in (8.2) and their ratio, and the SNR values achieved by applying our decomposition approach in the $\mathrm{CNC}$ and $\mathrm{TV}$ versions. In particular, for $\mathrm{CNC}$ model the parameter $\eta$ has been estimated according to the proposed automatic selection strategy, i.e. by using (8.4) and then (8.7)-(8.8), whereas the reported SNR values for TV are the highest achievable by letting $\eta$ varying in its domain $(0,1)$.

The impact of the mesh scale on decomposition results can be deduced from the SNR values reported in the fourth columns related to $S_{3}$ of Table 9.1, obtained by scaling $S_{3}$ to four different sphere radii $r \in\{1,2,4,10\}$. We observe that the mesh scale seems not to influence the quality of the achieved results for both CNC and TV. This can be explained by observing that when the mesh scale - represented by $h_{\min }$ - increases, the gradient norms of fields $v$ and $w$ are both downscaled by the same factor, so that the ratio $m / M$ remains constant.

On the other hand, the increasing of the mesh resolution, i.e. $S_{1}-S_{4}$ for $r=1$, and its structure i.e. $S_{3}-S_{4}$ for $r=1$, affect the input scalar field quality and the factor $1 / h$ which appears in the computation of the gradient magnitude. Therefore, for fixed radius $r=1$, the height $h$ decreases, and we observe an increasing performance, mainly caused both by the sampling quality and by improved gradient separability, due to the factor $1 / h$ in the gradient computation.

Finally, we notice from SNR values in Table 9.1 that CNC outperforms TV also in this scalar fields decomposition test. This is supported by the visual inspection of the decomposition results shown in Fig. 9.6, where one can observe, for the TV case, traces of the original cartoon component in the estimated smooth component $w^{*}$.

9.3. Example 3. In this last example we carry out a broader evaluation of the proposed CNC model - with $\tau_{c}=0.99$ and $\eta$ automatically selected by (8.4) and (8.7)-(8.8) - by applying it to the decomposition of noiseless and also noisy scalar fields defined over three different meshed surfaces referred to as sphere, vase and horse 


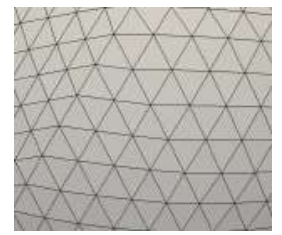

$S_{1}$

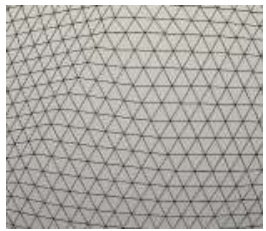

$S_{2}$

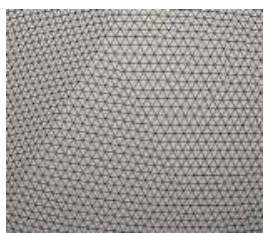

$S_{3}$

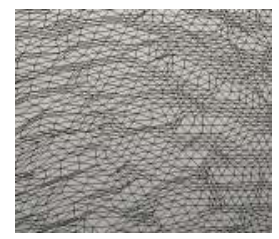

$S_{4}$

FIG. 9.5. Example 2: details of the meshes considered. $S_{1}-S_{3}$ represent different resolutions of structured mesh, while $S_{4}$ is an unstructured mesh with resolution similar to $S_{3}$.

\begin{tabular}{|c|c|c|c|c|c|c|c|}
\hline & $S_{1}$ & $S_{2}$ & $S_{3}$ & $S_{3}$ & $S_{3}$ & $S_{3}$ & $S_{4}$ \\
\hline$r$ & 1 & 1 & 1 & 2 & 4 & 10 & 1 \\
\hline$h_{\min }$ & $3.47 \mathrm{e}-2$ & $1.74 \mathrm{e}-2$ & $8.68 \mathrm{e}-3$ & $1.74 \mathrm{e}-2$ & $3.47 \mathrm{e}-2$ & $8.68 \mathrm{e}-2$ & $1.02 \mathrm{e}-3$ \\
\hline$e_{\max }(B)$ & $3.4 \mathrm{e}+3$ & $1.4 \mathrm{e}+4$ & $5.5 \mathrm{e}+4$ & $1.4 \mathrm{e}+4$ & $3.4 \mathrm{e}+3$ & $5.5 \mathrm{e}+2$ & $3.5 \mathrm{e}+4$ \\
\hline$a$ & $9.2 \mathrm{e}-1$ & $4.9 \mathrm{e}-1$ & $2.5 \mathrm{e}-1$ & $5.1 \mathrm{e}-1$ & 1.02 & 2.54 & 1.01 \\
\hline$M$ & 0.50 & 0.50 & 0.50 & 0.25 & 0.13 & 0.05 & 0.50 \\
\hline$m$ & 3.79 & 7.55 & 15.09 & 7.54 & 3.77 & 1.51 & 3.40 \\
\hline$m / M$ & 7.58 & 15.10 & 30.16 & 30.16 & 30.16 & 30.16 & 6.80 \\
\hline $\mathrm{CNC}$ & & & & & & & \\
\hline $\operatorname{SNR}\left(v^{*}\right)$ & 17.50 & 23.61 & 29.66 & 29.72 & 29.74 & 29.78 & 33.81 \\
\hline $\operatorname{SNR}\left(w^{*}\right)$ & 16.19 & 22.29 & 28.33 & 28.39 & 28.41 & 28.45 & 31.54 \\
\hline TV & & & & & & & \\
\hline $\operatorname{SNR}\left(v^{*}\right)$ & 13.95 & 20.41 & 26.47 & 26.47 & 26.47 & 26.47 & 26.61 \\
\hline $\operatorname{SNR}\left(w^{*}\right)$ & 12.65 & 19.08 & 25.14 & 25.14 & 25.14 & 25.14 & 24.34 \\
\hline
\end{tabular}

Example 2: properties of the sphere meshes $S_{1}-S_{4}$ and $S N R$ values for the CNC and TV models.

and characterized by resolutions $\mid$ sphere $|=(65538,131072)$,$| vase \mid=(52028,131072)$ and $\mid$ horse $\mid=(129218,258432)$. The sphere mesh coincides with mesh $S_{3}$ introduced in the previous example and also the considered field defined on it is the same as the one defined in that example.

First, we applied our CNC model in the noiseless case, i.e. for decomposing the two noise-free fields $u$ shown in the leftmost column of Figs. 9.8, 9.10. The very high performance of our approach in this test is evident from results in Fig. 9.7, where we report the estimated components $v^{*}$ and $w^{*}$ together with the associated SNR values.

Next, the noiseless fields defined over the three meshes vase, sphere and horse have been corrupted by additive white Gaussian noise of increasing standard deviations $\sigma \in\left\{1 \times 10^{-2}, 2 \times 10^{-2}, 3 \times 10^{-2}\right\}$, respectively. The noisy fields $b$, the noiseless fields $u$, and ground-truth cartoon and smooth components $v, w$ are shown in the leftmost columns of Figs. 9.8-9.10, whereas in the other two columns of the same figures we report the decomposition results obtained by our approach for two different values of the model parameter $\lambda$, together with the associated SNR values.

From the results in Figs. 9.8-9.10 we notice that for the smaller $\lambda$ values the proposed model acts as a good denoiser - in fact, $\operatorname{SNR}\left(u^{*}\right)$ values are high - whereas for the larger $\lambda$ values the quality of $\left\{v^{*}, w^{*}\right\}$ decompositions improves. In particular, the estimated cartoon components $v^{*}$ appear completely noise-free, while a fraction of the noise components are still visible in $w^{*}$. This behavior is more evident in 

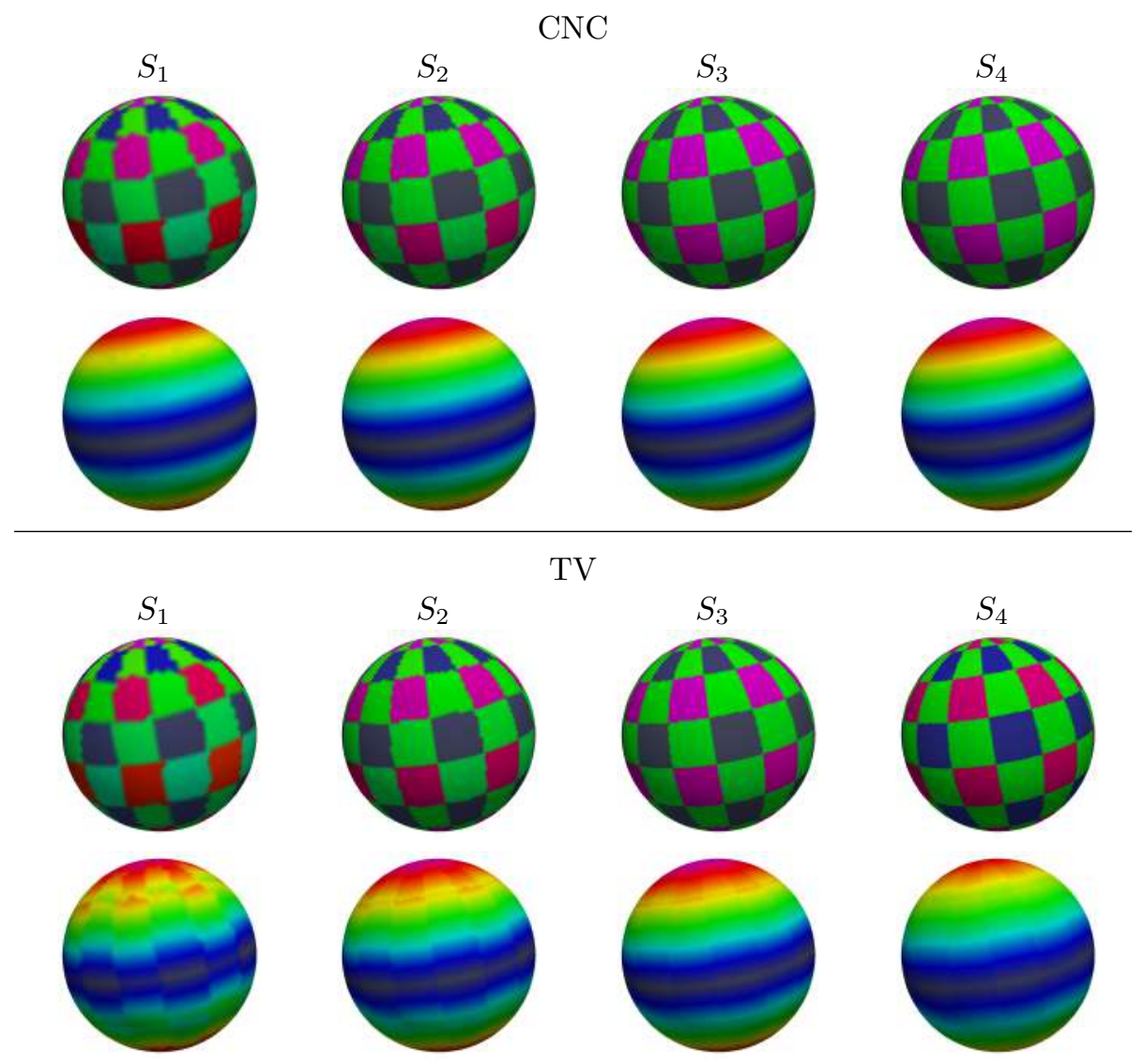

FIG. 9.6. Example 2: robustness to resizing the mesh resolution and structure for $S_{1}-S_{4}$ with radius $r=1$. The corresponding SNR values are reported in Table 9.1.

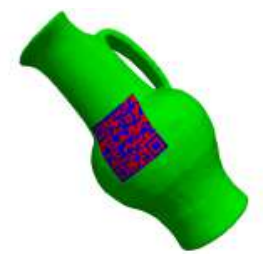

$$
\operatorname{SNR}\left(v^{*}\right)=51.68
$$

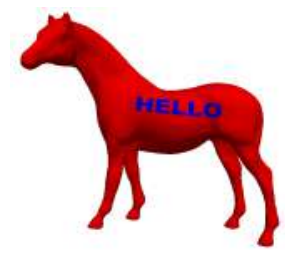

$\operatorname{SNR}\left(v^{*}\right)=52.25$

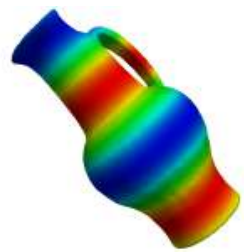

$\operatorname{SNR}\left(w^{*}\right)=50.33$

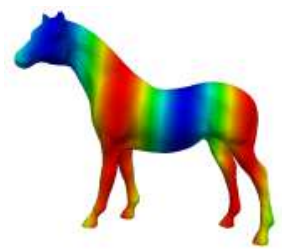

$\operatorname{SNR}\left(w^{*}\right)=63.31$

FIG. 9.7. Example 3: piecewise constant $\left(v^{*}\right)$ and smooth $\left(w^{*}\right)$ components estimated by our CNC decomposition approach applied to noisy scalar fields defined over the vase and horse meshed surfaces. Ground-truth fields are shown in Fig. 1.1 for vase and Fig. 9.10 for horse.

Fig. 9.10 corresponding to the test characterized by the highest noise level. This is explained by the fact that as the noise level increases the separability of the smooth and noise components becomes increasingly difficult. This suggests an effective twophase procedure which holds the potential for simultaneously achieving very good denoising and decomposition of noisy scalar fields on surfaces, visually illustrated in Fig. 9.11. In particular, in the first phase we compute a $\left\{v^{*}, w^{*}\right\}$ decomposition of the noisy observation $b=v+w+\epsilon$ by using our CNC model with a very large $\lambda$ value or, 
equivalently, the model variant (S3) outlined in Sect. 6. This should allow to capture in $v^{*}$ a very good estimate of the true cartoon component $v$, such that $w^{*}$ is also a very good estimate of the true noise-corrupted smooth component $w+\epsilon$. Hence, as a second phase we apply the model variant (S1) in Sect. 6 to $w^{*}$ obtained in the first phase so as to get good estimates $\bar{w}^{*}, \epsilon^{*}$ of the true smooth and noise components $w$, $\epsilon$. Clearly, if one is only interested in denoising the observed field $b$, then it can finally get a good estimate of the true noiseless field $u=v+w$ by summing $v^{*}$ output of the first phase with $\bar{w}^{*}$ output of the second phase, as illustrated in Fig. 9.11.

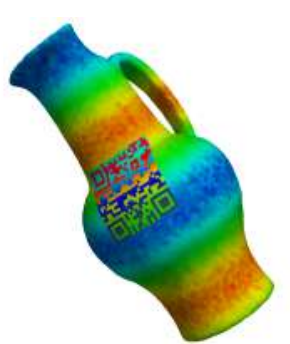

b

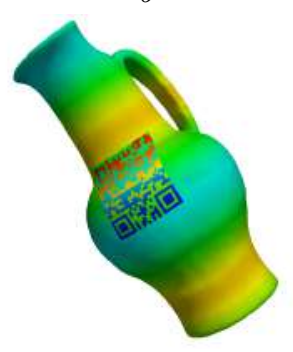

$u$

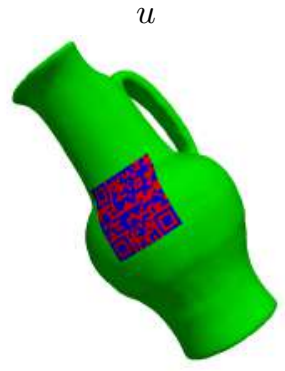

$v$

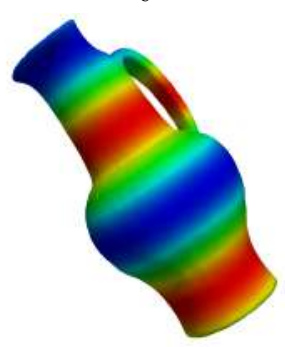

$w$

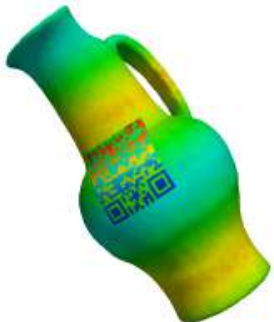

$\operatorname{SNR}\left(u^{*}\right)=23.88$
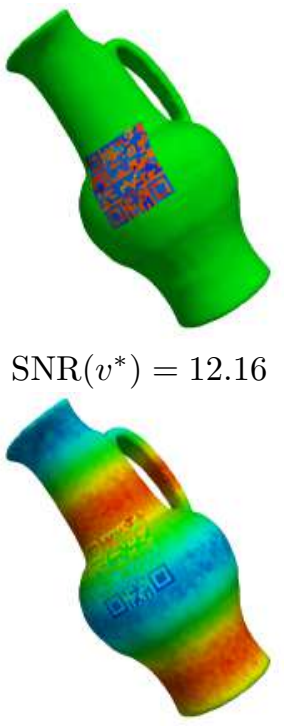

$\operatorname{SNR}\left(w^{*}\right)=10.88$

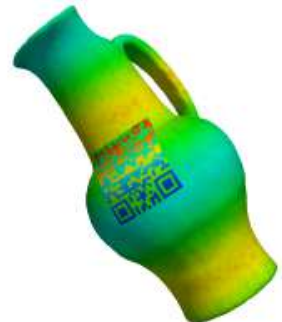

$\operatorname{SNR}\left(u^{*}\right)=22.36$

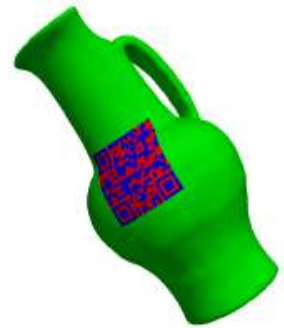

$\operatorname{SNR}\left(v^{*}\right)=32.31$

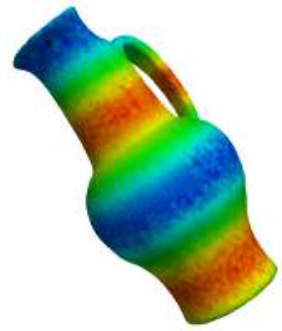

$\operatorname{SNR}\left(w^{*}\right)=18.64$

FIG. 9.8. Example 3: original scalar field components, at the top the noisy input b, with $\operatorname{SNR}(b)=21.83$ and $\sigma=10^{-2}$ (first column); decomposition results obtained for estimated $\eta$ parameter and $\lambda=10$ (second column) and $\lambda=80$ (third column) 


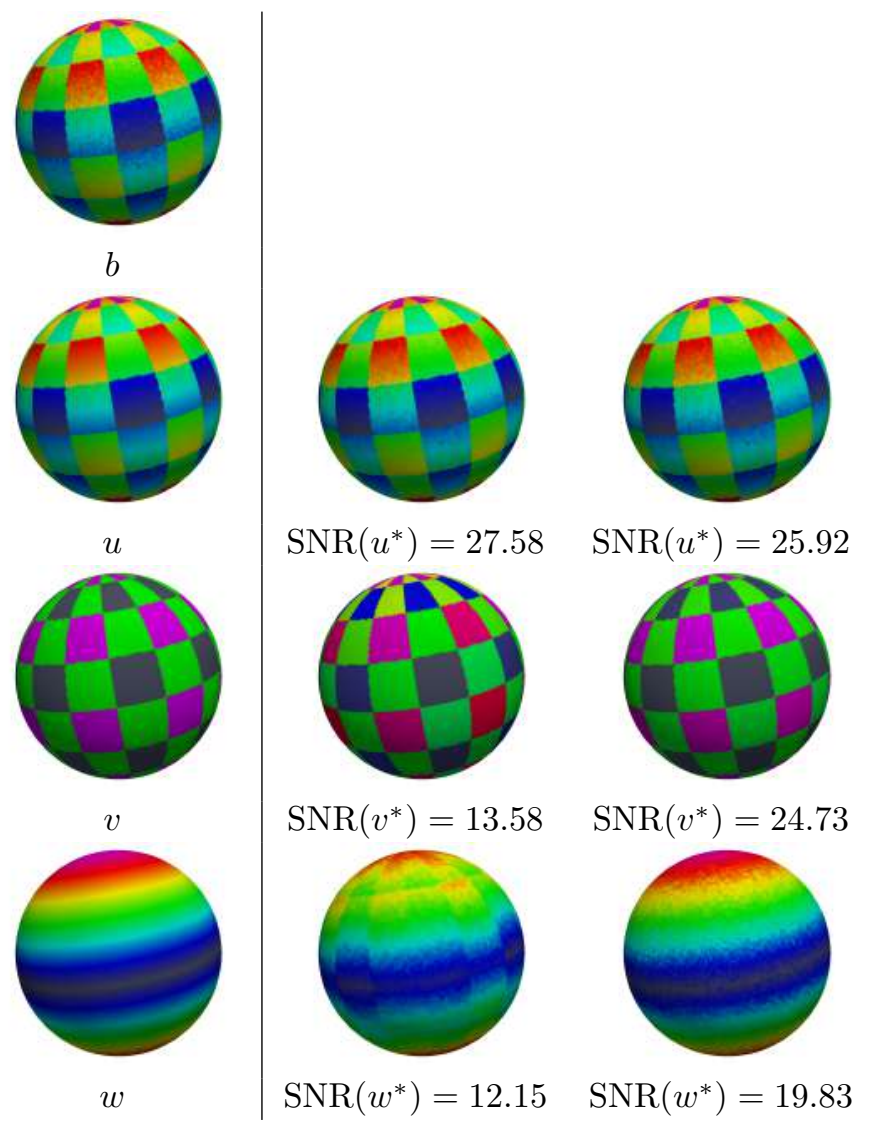

FIG. 9.9. Example 3: original scalar field components, at the top the noisy input b, with $\operatorname{SNR}(b)=21.33$ and $\sigma=2 \times 10^{-2}$ (first column); decomposition results obtained for estimated $\eta$ parameter and $\lambda=140$ (second column), $\lambda=10^{5}$ (third column)

10. Conclusions. This work enlarges the class of CNC variational approaches and can be used to denoise as well as to decompose noisy scalar fields defined over triangulated surfaces into piecewise constant and smooth components. The introduced energy functional is a linear mixture of a Tikhonov regularization term for the smooth component, a TV-like non-convex regularization term for the cartoon component and a quadratic fidelity term accounting for noise. The non-convex term is parametrized such that the free scalar parameter allows to tune its degree of non-convexity and, hence, to promote sparsity of gradient norms of the cartoon component more strongly than classical convex TV without renouncing to convexity of the total model. A selection strategy for the free model parameters has been presented, theoretically motivated and experimentally validated. An efficient ADMM algorithm has been proposed to compute the (unique) solution of our CNC model. Numerical experiments on images as well as functions on surfaces indicate the high quality of decompositions obtainable by our proposal, confirm the CNC strategy as a very valuable alternative to both the purely convex and purely non-convex approaches and prove the robustness of the proposed parameter selection strategy.

Future work will be devoted to improve our proposal both in the model and in the parameter selection perspective, namely we will investigate more powerful fidelity 


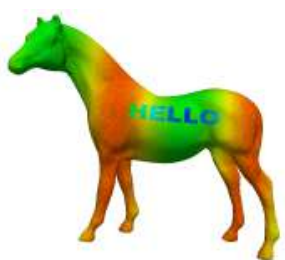

$b$

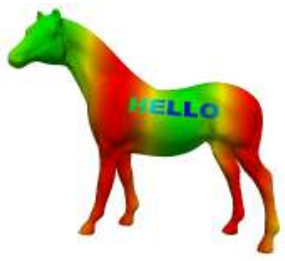

$u$

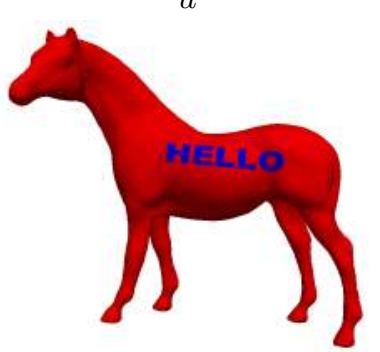

$v$

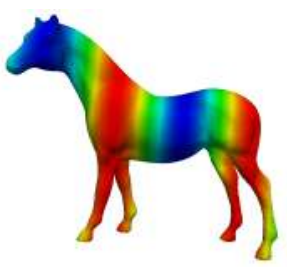

$w$

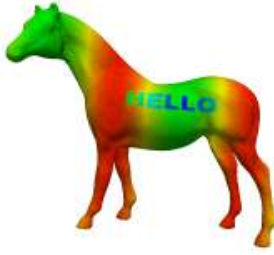

$\operatorname{SNR}\left(u^{*}\right)=24.71$

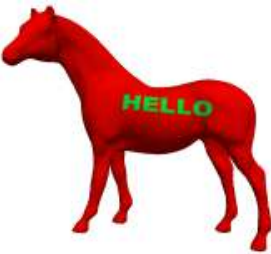

$\operatorname{SNR}\left(v^{*}\right)=7.28$

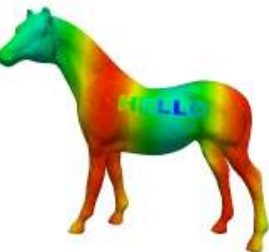

$\operatorname{SNR}\left(w^{*}\right)=18.87$

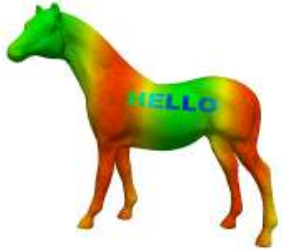

$\operatorname{SNR}\left(u^{*}\right)=19.78$

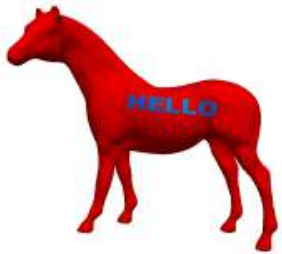

$\operatorname{SNR}\left(v^{*}\right)=17.76$

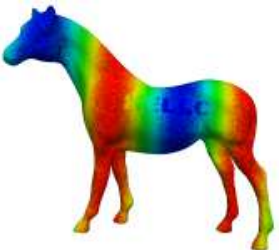

$\operatorname{SNR}\left(w^{*}\right)=18.90$

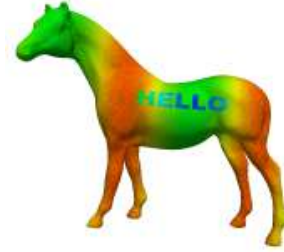

$\operatorname{SNR}\left(u^{*}\right)=16.04$

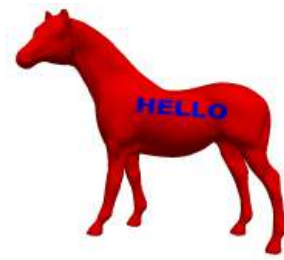

$\operatorname{SNR}\left(v^{*}\right)=29.63$

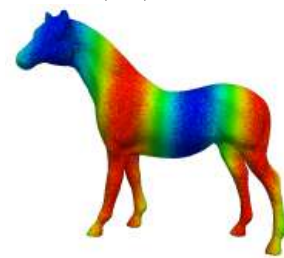

$\operatorname{SNR}\left(w^{*}\right)=15.57$

Fig. 9.10. Example 3: original scalar field components, at the top the noisy input b, with $\operatorname{SNR}(b)=16.04$ and $\sigma=3 \times 10^{-2}$ (first column); decomposition results obtained for estimated $\eta$ parameter and $\lambda=3$ (second column), $\lambda=100$ (third column), $\lambda=10^{12}$ (fourth column).

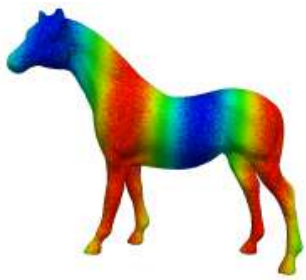

$b=w^{*}$

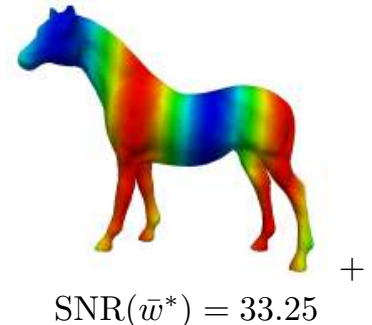

$\operatorname{SNR}\left(\bar{w}^{*}\right)=33.25$

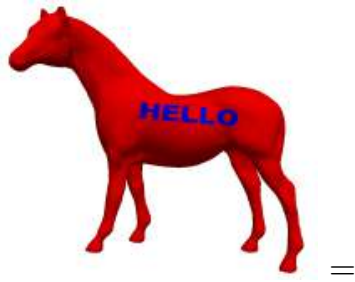

$v^{*}$

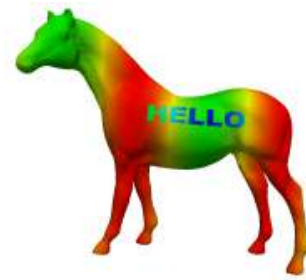

$\operatorname{SNR}\left(u^{*}\right)=33.39$

Fig. 9.11. Example 3: two-phases approach. From left to right: input $b$ to the second phase coinciding with output $w^{*}$ of the first phase shown in the last column of Fig. 9.10 with $\operatorname{SNR}(b)=$ 15.57; resulting $\bar{w}^{*}$ via the second phase; output $v^{*}$ of the first phase depicted in the right-most column of Fig. 9.10; reconstructed noise-free field $u^{*}=v^{*}+\bar{w}^{*}$. 
terms enforcing whiteness of the residual (see, e.g., [8]), which should allow for better separation of noise/smooth components, and we will investigate automatic methods for estimating quantities $m$ and $M$ in (8.2) also in noisy cases.

\section{REFERENCES}

[1] J.F.Aujol, G. Gilboa, T. Chan and S. Osher, Structure-Texture Image DecompositionModeling, Algorithms, and Parameter Selection, International Journal of Computer Vision, 2006, 67(1), 111-136.

[2] A. Chambolle, P.L. Lions, Image recovery via total variation minimization and related problems, Numer. Math., 76, 1997, 167-188.

[3] S. Osher, A. Solé, L. Vese, Image decomposition and restoration using total variation minimization and the $H^{1}$ norm, Multiscale Model. Simul., 1(3), 2003, 349-370.

[4] L. Vese, S. Osher, Modeling textures with total variation minimization and oscillating patterns in image processing, J. Sci. Comput., 19(13), 2003, 553-572.

[5] A. Gholami, S.M. Hosseini, A balanced combination of Tikhonov and total variation regularizations for reconstruction of piecewise-smooth signals, Signal Processing, 2013, 93(7) : $1945-1960$.

[6] Y. Meyer, Oscillating Patterns in Image Processing and Nonlinear Evolution Equations: The Fifteenth Dean Jacqueline B. Lewis Memorial Lectures, University Lecture Series, vol. 22, AMS, Providence, 2001.

[7] A. Blake, A. Zisserman, Visual Reconstruction, Cambridge, MA, USA: MIT Press, 1987.

[8] A. Lanza, S. Morigi, F. Sgallari, A.J. Yezzi, Variational image denoising based on autocorrelation whiteness, SIAM Journal on Imaging Sciences, 2013, 6(4) : 1931-1955.

[9] P.Y. Chen and I.W. Selesnick, Group-sparse signal denoising: non-convex regularization, convex optimization, IEEE Trans. on Sign. Proc., 2014, 62 : 3464-3478.

[10] A. Lanza, S. Morigi, and F. Sgallari, Convex image denoising via non-convex regularization, Scale Space and Variational Methods in Computer Vision, 2015, 9087 : 666-677.

[11] A. Lanza, S. Morigi, F. Sgallari, Convex image denoising via non-convex regularization with parameter selection, Journal of Mathematical Imaging and Vision, vol. 56(2), pp. 195-220, 2016.

[12] A. Lanza, S. Morigi, I. Selesnick, F. Sgallari, Nonconvex nonsmooth optimization via convexnonconvex majorization-minimization, Numerische Mathematik, vol. 136(2), pp. 343-381, 2017.

[13] C. W. Lu, Image restoration and decomposition using nonconvex nonsmooth regularisation and negative Hilbert-Sobolev norm, IET Image Processing, 2012, textbf6(6) : 706-716.

[14] M. Nikolova, Estimation of Binary Images by Minimizing Convex Criteria, in Proc. IEEE Int. Conf. Image Process., 2, 108-112, 1998.

[15] A. Parekh, I.W. Selesnick, Convex Denoising using Non-Convex Tight Frame Regularization arXiv preprint arXiv:1504.00976, 2015

[16] L.I. Rudin, S. Osher and E. Fatemi, Nonlinear total variation based noise removal algorithms, Physics D, 1992, 60(1-4) : 259-268.

[17] I. W. Selesnick, I. Bayram, Sparse signal estimation by maximally sparse convex optimization, IEEE Trans. on Signal Proc., 2014, 62(5) : 1078-1092.

[18] I. W. Selesnick, A. Parekh, and I. Bayram, Convex 1-D total variation denoising with non-convex regularization, IEEE Signal Proc. Letters, 2015, 22(2) : 141-144.

[19] M. Botsch, L. Kobbelt, M. Pauly, P. Alliez, B. Levy, Polygon Mesh Processing Ed. A. K. Peters, CRC Press, 2010.

[20] G. Gilboa, A Total Variation Spectral Framework for Scale and Texture Analysis, SIAM Journal on Imaging Sciences, 2014, 7(4) : 1937-1961.

[21] D. Horesh, and G. Gilboa, Separation Surfaces in the Spectral TV Domain for Texture Decomposition, IEEE Transaction on Image Processing, 2016, 25(9) : 4260-4270.

[22] C.-H. Zhang, Nearly unbiased variable selection under minimax concave penalty, Annals of Statistics, 2010, 38(2) : 894-942.

[23] R. Chan, A. Lanza, S. Morigi, F. Sgallari, Convex non-convex image segmentation, Numerische Mathematik, 2018, 138(3) : 635-680.

[24] N.L. Tsitsas, On block matrices associated with discrete trigonometric transforms and their use in the theory of wave propagation, J. of Comp. Math., 2010, 6: 864-878.

\section{Appendix. Proof of Proposition 5.2.}


Proof. First, we rewrite the function $\mathcal{J}_{1}$ in (5.4) as follows:

$$
\begin{aligned}
\mathcal{J}_{1}(v, w ; \lambda, \eta, \gamma) & =\frac{\lambda}{2}\left(v^{T} S_{V} v+w^{T} S_{V} w+2 v^{T} S_{V} w\right)+\frac{\eta}{2} w^{T} D^{T} S D w-\frac{\gamma}{2} v^{T} D^{T} S D v \\
& =\frac{1}{2}\left(v^{T} w^{T}\right)\left(\begin{array}{ll}
\lambda S_{V}-\gamma D^{T} S D & \lambda S_{V} \\
\lambda S_{V} & \lambda S_{V}+\eta D^{T} S D
\end{array}\right)\left(\begin{array}{c}
v \\
w
\end{array}\right)
\end{aligned}
$$

The function $\mathcal{J}_{1}$ in (10.1) is quadratic in $(v, w)$, hence it is convex in $(v, w)$ if and only if its Hessian $H_{\mathcal{J}_{1}} \in \mathbb{R}^{2 n_{V} \times 2 n_{V}}$ is at least positive semidefinite, that is if and only if

$$
H_{\mathcal{J}_{1}}=\left(\begin{array}{cc}
S_{V}^{1 / 2} & 0 \\
0 & S_{V}^{1 / 2}
\end{array}\right)\left(\begin{array}{ll}
\lambda I_{n}-\gamma B & \lambda I_{n} \\
\lambda I_{n} & \lambda I_{n}+\eta B
\end{array}\right)\left(\begin{array}{cc}
S_{V}^{1 / 2} & 0 \\
0 & S_{V}^{1 / 2}
\end{array}\right) \succeq 0,
$$

with matrix $B \in \mathbb{R}^{n_{V} \times n_{V}}$ defined in (5.6). Since $B$ is symmetric and positive semidefinite, it admits the eigenvalue decomposition

$$
B=V^{T} E V, \quad E=\operatorname{diag}\left(e_{1}, e_{2}, \ldots, e_{n_{V}}\right), \quad V^{T} V=V V^{T}=I_{n_{V}},
$$

with $e_{1} \geq e_{2} \geq \ldots \geq e_{n_{V}} \geq 0$ indicating the real non-negative eigenvalues of $B$. Replacing (10.3) into (10.2), we obtain:

$$
H_{\mathcal{J}_{1}}=\left(\begin{array}{cc}
S_{V}^{1 / 2} V^{T} & 0 \\
0 & S_{V}^{1 / 2} V^{T}
\end{array}\right) \underbrace{\left(\begin{array}{cc}
\Lambda_{1,1} & \Lambda_{1,2} \\
\Lambda_{2,1} & \Lambda_{2,2}
\end{array}\right)}_{\Lambda}\left(\begin{array}{cc}
V S_{V}^{1 / 2} & 0 \\
0 & V S_{V}^{1 / 2}
\end{array}\right) \succeq 0,
$$

where the blocks $\Lambda_{1,1}, \Lambda_{1,2}, \Lambda_{2,1}, \Lambda_{2,2} \in \mathbb{R}^{n_{V} \times n_{V}}$ are diagonal matrices defined by

$$
\Lambda_{1,1}=\lambda I_{n_{V}}-\gamma E, \quad \Lambda_{2,2}=\lambda I_{n_{V}}+\eta E, \quad \Lambda_{1,2}=\Lambda_{2,1}=\lambda I_{n_{V}} .
$$

Following [24], there exists a permutation matrix $P \in \mathbb{R}^{2 n_{V} \times 2 n_{V}}$ such that

$$
P \Lambda P^{T}=\Lambda^{\prime}=\operatorname{diag}\left(\Lambda_{1}^{\prime}, \Lambda_{2}^{\prime}, \ldots, \Lambda_{n_{V}}^{\prime}\right),
$$

with matrices $\Lambda_{k}^{\prime} \in \mathbb{R}^{2 \times 2}$ defined by

$$
\left[\Lambda_{k}^{\prime}\right]_{i, j}=\left[\Lambda_{i, j}\right]_{k}, \quad k \in\left\{1, \ldots, n_{V}\right\}, \quad i, j \in\{1,2\} .
$$

Thus, the original $2 \times 2$ block matrix $\Lambda$ in (10.4) with diagonal blocks $\Lambda_{i, j}$ of order $n_{V}$ can be transformed into the block-diagonal matrix $\Lambda^{\prime}$ with $n_{V}$ full blocks $\Lambda_{k}^{\prime}$ of order 2 which, in accordance with (10.5) and (10.7), take the form

$$
\Lambda_{k}^{\prime}=\left(\begin{array}{ll}
\lambda-\gamma e_{k} & \lambda \\
\lambda & \lambda+\eta e_{k}
\end{array}\right), \quad k \in\left\{1, \ldots, n_{V}\right\} .
$$

The real symmetric matrices $\Lambda_{k}^{\prime}$ in (10.8) admit the eigenvalue decompositions

$$
\Lambda_{k}^{\prime}=R_{k}^{T} L_{k} R_{k}, \quad L_{k}=\operatorname{diag}\left(l_{k}^{(1)}, l_{k}^{(2)}\right), \quad R_{k}^{T} R_{k}=R_{k} R_{k}^{T}=I_{2}, \quad k \in\left\{1, \ldots, n_{V}\right\},
$$

with $l_{k}^{(1)}, l_{k}^{(2)}$ the two real eigenvalues of $\Lambda_{k}^{\prime}$. Recalling (10.6) and introducing the block diagonal matrix $R:=\operatorname{diag}\left(R_{1}, \ldots, R_{n_{V}}\right) \in \mathbb{R}^{2 n_{V} \times 2 n_{V}}$, which is orthogonal as blocks $R_{k}$ are all orthogonal, condition in (10.4) can be equivalently rewritten as follows:

$$
H_{\mathcal{J}_{1}}=W^{T} L W \succeq 0 \quad \text { with } \quad W=R P\left(\begin{array}{cc}
V S_{V}^{1 / 2} & 0 \\
0 & V S_{V}^{1 / 2}
\end{array}\right) \in \mathbb{R}^{2 n_{V} \times 2 n_{V}}
$$


a full rank matrix and $L \in \mathbb{R}^{2 n_{V} \times 2 n_{V}}$ the diagonal matrix containing all the eigenvalues of all the matrices $\Lambda_{k}^{\prime}$ in (10.8), namely

$$
L=\operatorname{diag}\left(l_{1}, l_{2}, \ldots, l_{2 n_{V}}\right)=\operatorname{diag}\left(l_{1}^{(1)}, l_{1}^{(2)}, l_{2}^{(1)}, l_{2}^{(2)}, \ldots, l_{n_{V}}^{(1)}, l_{n_{V}}^{(2)}\right) .
$$

In particular, the pair of eigenvalues $l_{k}^{(1)}, l_{k}^{(2)}$ of matrix $\Lambda_{k}^{\prime}$ in (10.8) is given by

$$
l_{k}^{(1),(2)}=\lambda+\frac{\eta-\gamma}{2} e_{k} \pm \sqrt{\lambda^{2}+\left(\frac{\eta+\gamma}{2} e_{k}\right)^{2}} .
$$

Condition in (10.10) is satisfied if and only if matrix $L$ in (10.11) is positive semidefinite, that is if and only if all its diagonal entries in (10.12) are non-negative. Since such entries coincide with the eigenvalues of matrices $\Lambda_{k}^{\prime}$ in (10.8), function $\mathcal{J}_{1}$ is convex if and only if all matrices $\Lambda_{k}^{\prime}$ are positive semidefinite. According to Sylvester's criterion, this happens if and only if

$$
\left\{\begin{array}{rl}
\lambda-\gamma e_{k} & \geq 0 \\
\lambda+\eta e_{k} & \geq 0 \\
\left(\lambda-\gamma e_{k}\right)\left(\lambda+\eta e_{k}\right)-\lambda^{2} & \geq 0
\end{array} \Longrightarrow \gamma \leq \frac{\lambda \eta}{\lambda+\eta e_{k}} \quad \forall k \in\left\{1, \ldots, n_{V}\right\},\right.
$$

hence condition (5.5) follows.

To complete the proof, first we recall that, based on (10.10), we can write

$$
\mathcal{J}_{1}(x ; \lambda, \eta, \gamma)=\frac{1}{2} x^{T} H_{J_{1}} x=\frac{1}{2} x^{T} W^{T} L W x, \quad x=(v ; w),
$$

with $W \in \mathbb{R}^{2 n_{V} \times 2 n_{V}}$ in (10.10) a full-rank matrix and $L \in \mathbb{R}^{2 n v \times 2 n_{V}}$ the diagonal matrix defined in (10.11)-(10.12). We now analyze $L$. The diagonal entries of $L$ depend on the parameters $\lambda, \eta, \gamma$ as well as on the eigenvalues $e_{k}, k=1, \ldots, n_{V}$, of matrix $B \in \mathbb{R}^{n_{V} \times n_{V}}$ defined in (5.6). It is easy to prove that $B$ is always symmetric positive semidefinite with rank equal to $n_{V}-1$, in fact:

1) $B=A^{T} A$ with $A:=S^{1 / 2} D S_{V}^{-1 / 2}$, hence $B$ is symmetric positive semidefinite and has the same rank of $A$;

2) $S^{1 / 2}, S_{V}^{-1 / 2}$ are full rank square matrices, hence $A$ has the same rank of $D$;

3) $D$ discretizes the gradient operator, hence its null-space has dimension 1.

This implies that the eigenvalues of $B$ satisfy $e_{1} \geq e_{2} \geq \ldots \geq e_{n_{V}-1}>e_{n_{V}}=0$. It follows from (10.12) that the $n_{V}$-th pair of diagonal entries of $L$ - obtained by replacing $e_{k}=e_{n_{V}}=0$ in $(10.12)$ - is given by $\left(l_{n_{V}}^{(1)}, l_{n_{V}}^{(2)}\right)=(2 \lambda, 0)$, hence $L$ has rank less than or equal to $2 n_{V}-1$, independently of parameters $\lambda, \eta, \gamma$. In particular, it is easy to prove that if $\gamma \geq \eta /\left(1+\eta e_{\max } / \lambda\right)$, then $L$ can have rank less than $2 n_{V}-1$. Instead, if $\gamma<\eta /\left(1+\eta e_{\max } / \lambda\right)$, then $L$ has rank $2 n_{V}-1$. In fact, the remaining $n_{V}-1$ pairs of eigenvalues $\left(l_{k}^{(1)}, l_{k}^{(2)}\right)$ of $L$ obtained by replacing in (10.12) the positive eigenvalues $e_{k}, k=1, \ldots, n_{V}-1$, of $B$, are positive if and only if all the $n_{V}-1$ matrices $\Lambda_{k}^{\prime}$, $k=1, \ldots, n_{V}-1$, defined in (10.8) are positive definite. According to Sylvester's criterion, and following analogous derivations as in (10.13), it is immediate to prove that this happens if and only if $\gamma<\eta /\left(1+\eta e_{\max } / \lambda\right)$.

Hence, if inequality (5.5) holds in strict sense, then $L$ is positive semidefinite and has rank equal to $2 n_{V}-1$, such that its diagonal entries are all positive but one which is zero. This means that there exists one and only one unit-norm vector $\bar{d} \in \mathbb{R}^{2 n_{V}}$ such that $\mathcal{J}_{1}$ assumes constant null values along the homogeneous straight line $x=t \bar{d}$, 
$t \in \mathbb{R}$. It comes easily from the original expression of $\mathcal{J}_{1}$ in (5.4) that such unit-vector $\bar{d}$ coincides with $d$ defined in (5.1). Hence, $\mathcal{J}_{1}$ is strongly convex over any feasible set defined by a linear constraint of the form in (5.2).

\section{Proof of Proposition 7.1.}

Proof. Condition (7.15) comes easily from Proposition 2 in [9] and for the proof of statement (7.17) we refer the reader to Proposition 4.5 in [11].

We now prove statement (7.18). First, we notice that if $\|r\|_{2}=0$, then the solution of (7.16) is clearly $z^{*}=0$. We now consider the case $\|r\|_{2}>0$. Based on statement (7.17), by setting $z=\xi r, \xi \geq 0$, we turn the original unconstrained 3-dimensional problem in (7.16) into the following equivalent constrained 1-dimensional problem:

$$
\xi^{*}=\arg \min _{0 \leq \xi \leq 1}\left\{f(\xi):=\phi\left(\|r\|_{2} \xi ; a\right)+\frac{\omega}{2}\|r\|_{2}^{2}\left(\xi^{2}-2 \xi\right)\right\},
$$

where we omitted the constants and introduced the function $f: \mathbb{R}_{+} \rightarrow \mathbb{R}$. Since $\phi$ is continuously differentiable on $\mathbb{R}_{+}$- see Sect. 2 - the function $f$ in (10.15) is also continuously differentiable on $\mathbb{R}_{+}$. Moreover, $f$ is strictly convex as it is the restriction of the strictly convex function $\theta$ in (7.14) to the half-line $\xi r, \xi \geq 0$. Hence, a necessary and sufficient condition for a point $0<\xi<1$ to be the global minimizer of $f$ is

$$
f^{\prime}(\xi)=0 \Longleftrightarrow\|r\|_{2}\left[\phi^{\prime}\left(\|r\|_{2} \xi ; a\right)+\omega\|r\|_{2}(\xi-1)\right]=0 .
$$

Since $f$ is continuously differentiable and strictly convex on $\mathbb{R}_{+}, f^{\prime}(\xi)$ is continuous and strictly increasing in the domain $0 \leq \xi \leq 1$ and at the extremes we have:

$$
\begin{aligned}
& f^{\prime}\left(0^{+}\right)=\|r\|_{2}\left[\sqrt{2 a}-\omega\|r\|_{2}\right] \geq 0 \text { for }\|r\|_{2} \leq \sqrt{2 a} / \omega \quad(<0 \text { otherwise }) \\
& f^{\prime}(1)=\|r\|_{2} \phi^{\prime}\left(\|r\|_{2} ; a\right) \quad>0 \text { for }\|r\|_{2}<\sqrt{2 / a} \quad \text { (=0 otherwise) }
\end{aligned}
$$

Moreover, since $\omega>a \Longrightarrow \sqrt{2 a} / \omega<\sqrt{2 / a}$, we have the three cases:

$$
\begin{array}{ll}
\|r\|_{2} \leq \sqrt{2 a} / \omega & \Longrightarrow f^{\prime}\left(0^{+}\right) \geq 0, f^{\prime}(1)>0 ; \\
\text { b) } \quad \sqrt{2 a} / \omega<\|r\|_{2}<\sqrt{2 / a} & \Longrightarrow f^{\prime}\left(0^{+}\right)<0, f^{\prime}(1)>0 ; \\
\text { c) } \sqrt{2 / a} \leq\|r\|_{2} & \Longrightarrow f^{\prime}\left(0^{+}\right)<0, f^{\prime}(1)=0 .
\end{array}
$$

For cases a) and c) the solution $\xi^{*}$ of (10.15) belongs to the boundary of the optimization interval $[0,1]$, namely $\xi^{*}=0$ for case a) and $\xi^{*}=1$ for case c). For case b), instead, $\xi^{*} \in(0,1)$ is the solution of the first-order optimality condition (10.16), i.e.

$$
\sqrt{2 a}-a\|r\|_{2} \xi+\omega\|r\|_{2}(\xi-1)=0 \Longleftrightarrow \xi^{*}=\left(\omega-\sqrt{2 a} /\|r\|_{2}\right) /(\omega-a) .
$$

The proof of statement (7.18) is thus completed.

\section{Proof of Proposition 8.1.}

Proof. Existence and uniqueness of the second intersection point - the first is the origin - comes easily form the definitions of $f_{v}, f_{w}$ in (8.3) and of $\phi$ in (2.1).

To prove (8.5), first we notice that the function $f_{v}$ in (8.3) is piecewise defined like the function $\phi$ - see $(2.1)$ - on the two sub-domains $[0, \sqrt{2 / a})$ and $[\sqrt{2 / a},+\infty)$. Denoting by $\hat{t}$ the (positive) abscissa of the intersection point between $f_{w}$ and the prolongation of the second piece of $f_{v}$ to $[0,+\infty)$ - namely, the horizontal half-line having intercept $(1-\eta)$ - we have

$$
\eta t^{2}=1-\eta \Longrightarrow \hat{t}=\sqrt{(1-\eta) / \eta} .
$$


In order for $\hat{t}$ above to be an acceptable solution, that is to represent the abscissa $\bar{t}$ of the actual intersection point for $t>0$ between $f_{v}$ and $f_{w}, \hat{t}$ must belong to the domain of definition $[\sqrt{2 / a},+\infty)$ of the second piece of $f_{v}$, namely:

$$
\begin{aligned}
\hat{t} \geq \sqrt{\frac{2}{a}} \Longleftrightarrow a \geq \frac{2 \eta}{1-\eta} \Longleftrightarrow \tau_{c} \frac{2 \eta}{1-\eta} \frac{1}{1+\eta e_{\max } / \lambda} \geq \frac{2 \eta}{1-\eta}(10.21) \\
\Longleftrightarrow \tau_{c} \geq 1+\eta \mu \Longleftrightarrow \eta \leq\left(\tau_{c}-1\right) / \mu,
\end{aligned}
$$

where in (10.21) we replaced the expression of $a$ as a function of $\lambda, \eta, \tau_{c}$ given in the convexity condition (5.12) and in (10.22) we substituted $\nu=e_{\max } / \lambda$. In case that (10.22) is not satisfied, then clearly $\bar{t}$ coincides with the abscissa of the intersection for $t>0$ between $f_{w}$ and the first piece of $f_{v}$. In particular, recalling the definitions of $f_{v}, f_{w}$ in (8.3) and of $\phi$ in (2.1), we have:

$$
\eta t^{2}=(1-\eta)\left(\sqrt{2 a} t-\frac{a}{2} t^{2}\right) \Longleftrightarrow \bar{t}=\frac{(1-\eta) \sqrt{2 a}}{\eta+(1-\eta) \frac{a}{2}} \Longleftrightarrow \bar{t}=\sqrt{\frac{1-\eta}{\eta}} \frac{2 \sqrt{C}}{1+C},
$$

where the last equality comes from replacing the expression of $a$ in (5.12) and then algebraically manipulating the result, with $C$ defined in (8.6).

To prove (8.7), first we denote by $\varrho\left(\eta ; \lambda, \tau_{c}\right):(0,1) \rightarrow \mathbb{R}_{+}^{*}$ the piecewise-defined function in (8.5) which, for any given pair of parameters $\left(\lambda, \tau_{c}\right) \in \mathbb{R}_{+}^{*} \times \mathbb{R}_{+}^{*}$, maps $\eta$ into the abscissa $\bar{t}$ and by $\varrho_{1}\left(\eta ; \lambda, \tau_{c}\right):\left(0, \eta_{12}\right] \rightarrow \mathbb{R}_{+}^{*}, \varrho_{2}\left(\eta ; \lambda, \tau_{c}\right):\left(\eta_{12}, 1\right) \rightarrow \mathbb{R}_{+}^{*}$ the two pieces of $\varrho$ defined in (8.5), with $\eta_{12}$ defined in (8.6). For any given pair $\left(\lambda, \tau_{c}\right) \in \mathbb{R}_{+}^{*} \times \mathbb{R}_{+}^{*}$, the functions $\varrho_{1}$ and $\varrho_{2}$ are clearly both continuously differentiable in $\eta$ over the domain $(0,1)$ and, in particular, we have $\varrho_{1}\left(\eta_{12} ; \lambda, \tau_{c}\right)=\varrho_{2}\left(\eta_{12} ; \lambda, \tau_{c}\right)=$ $\sqrt{\left(1-\eta_{12}\right) / \eta_{12}}$. Hence, the function $\varrho$ is continuous in $\eta$ over its the entire domain $(0,1)$. For what concerns the first-order derivatives with respect to $\eta$ of functions $\varrho_{1}$ and $\varrho_{2}$, we have:

$$
\begin{aligned}
& \frac{\partial \varrho_{1}\left(\eta ; \lambda, \tau_{c}\right)}{\partial \eta}=-\frac{1}{2 \eta^{2}} \sqrt{\frac{\eta}{1-\eta}}<0 \forall \eta \in(0,1), \\
& \frac{\partial \varrho_{2}\left(\eta ; \lambda, \tau_{c}\right)}{\partial \eta}=-\frac{1}{2 \eta^{2}} \sqrt{\frac{\eta}{1-\eta}} \frac{2 \sqrt{C}}{1+C}+\sqrt{\frac{1-\eta}{\eta}} \frac{1-C}{\sqrt{C}} \frac{\partial C}{\partial \eta}<0 \quad \forall \eta \in(0,1),
\end{aligned}
$$

where the last inequality in (10.25) can easily be proved after some algebra. It follows from (10.24)-(10.25) that the functions $\varrho_{1}, \varrho_{2}$, hence $\varrho$, are monotonically decreasing and, thus, invertible in the variable $\eta$. The inverse functions $\varrho_{1}^{-1}\left(\bar{t} ; \lambda, \tau_{c}\right), \varrho_{2}^{-1}\left(\bar{t} ; \lambda, \tau_{c}\right)$ can be obtained starting from (8.5):

$$
\begin{aligned}
& \bar{t}=\sqrt{\frac{1-\eta}{\eta}} \Longleftrightarrow \bar{t}^{2}=\frac{1-\eta}{\eta} \Longleftrightarrow \eta \bar{t}^{2}+\eta=1 \Longleftrightarrow \eta=\frac{1}{1+\bar{t}^{2}} \\
& \bar{t}=\sqrt{\frac{1-\eta}{\eta}} \frac{2 \sqrt{C}}{1+C} \Longleftrightarrow \bar{t}^{2}=4 \frac{1-\eta}{\eta} \frac{\tau_{c}(1+\eta \nu)}{\tau_{c}^{2}+(1+\eta \nu)^{2}},
\end{aligned}
$$

where the latter equation in (10.27) comes from the former after replacing the expression of $C$ in (8.6). Further simple algebraic manipulations yield to the fourth-degree polynomial equation in (8.7). 